珮 


\title{
Akten zur Auswärtigen Politik der Bundesrepublik Deutschland
}

Herausgegeben im Auftrag des Auswärtigen Amts vom Institut für Zeitgeschichte

\author{
Hauptherausgeber \\ Hans-Peter Schwarz
}

\author{
Mitherausgeber \\ Helga Haftendorn, Klaus Hildebrand, \\ Werner Link, Horst Möller und Rudolf Morsey
}

R. Oldenbourg Verlag München 2001 


\section{Akten zur Auswärtigen Politik der Bundesrepublik Deutschland 1953}

Band I: 1. Januar bis 30. Juni 1953

Wissenschaftliche Leiterin

Ilse Dorothee Pautsch

Bearbeiter

Matthias Jaroch und Mechthild Lindemann

R. Oldenbourg Verlag München 2001 
Die Deutsche Bibliothek - CIP-Einheitsaufnahme

\begin{abstract}
Akten zur auswärtigen Politik der Bundesrepublik Deutschland /
hrsg. im Auftr. des Auswärtigen Amts vom Institut für

Zeitgeschichte. - München : Oldenbourg.
\end{abstract}

1953.

Bd. 1. 1. Januar bis 30. Juni 1953. -2001

ISBN 3-486-56560-5

(C) 2001 Oldenbourg Wissenschaftsverlag GmbH, München

Rosenheimer Straße 145, D-81671 München

Telefon: (089) 45051-0, Internet: http://www.oldenbourg.de

Das Werk einschließlich aller Abbildungen ist urheberrechtlich geschützt. Jede Verwertung außerhalb der Grenzen des Urheberrechtsgesetzes ist ohne Zustimmung des Verlages unzulässig und strafbar. Dies gilt insbesondere für Vervielfältigungen, Übersetzungen, Mikroverfilmungen und die Einspeicherung und Bearbeitung in elektronischen Systemen.

Umschlaggestaltung: Dieter Vollendorf

Gedruckt auf säurefreiem, alterungsbeständigem Papier

Gesamtherstellung: R. Oldenbourg Graphische Betriebe GmbH, München

ISBN 3-486-56560-5 


\section{Inhalt}

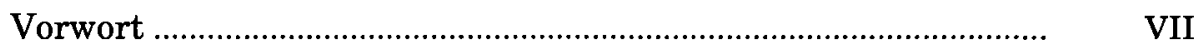

Vorbemerkungen zur Edition........................................................... VIII

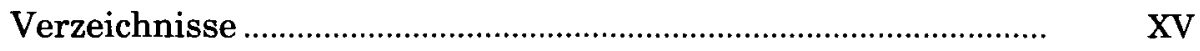

Dokumentenverzeichnis ................................................................ XVII

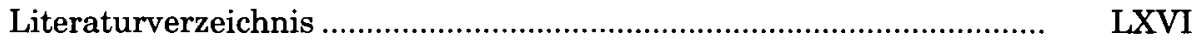

Abkürzungsverzeichnis ................................................................ LXXVIII

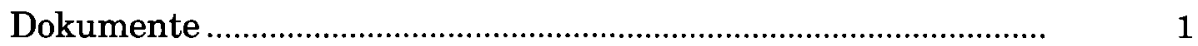

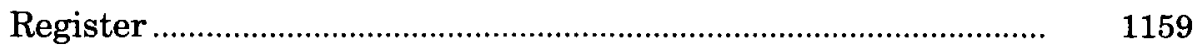

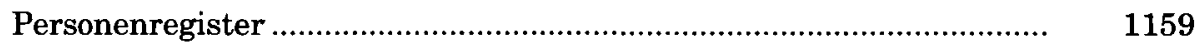

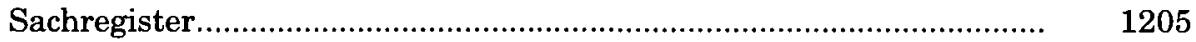

Anhang: Organisationsplan des Auswärtigen Amts vom Mai/Juni 1953 



\section{Vorwort}

Mit den „Akten zur Auswärtigen Politik der Bundesrepublik Deutschland 1953“ wird die Publikation von Dokumenten aus dem Politischen Archiv des Auswärtigen Amts über die Jahre 1949 bis 1962 fortgesetzt.

Das Erscheinen des vorliegenden Bandes gibt Anlaß, allen an dem Werk Beteiligten zu danken. So gilt mein verbindlichster Dank dem Auswärtigen Amt, insbesondere dem Politischen Archiv sowie den Damen und Herren in den Referaten, die beim Deklassifizierungsverfahren zur Offenlegung der Dokumente beigetragen haben. In gleicher Weise zu danken ist dem Bundesarchiv in Koblenz für die Erlaubnis, einige Aufzeichnungen aus dem Nachlaß des Botschafters a. D. Herbert Blankenhorn abdrucken zu können, sowie der Stiftung Bundeskanzler-Adenauer-Haus in Rhöndorf.

Besonderer Dank gebührt ferner den Kollegen im Herausgebergremium, die sich ihrer viel Zeit in Anspruch nehmenden Aufgabe in bewährter Kollegialität gewidmet haben. Ferner sei die tadellose Zusammenarbeit mit den zuständigen Persönlichkeiten und Gremien des Instituts für Zeitgeschichte dankbar hervorgehoben. Gedankt sei auch dem präzise arbeitenden Verlag R. Oldenbourg.

Das Hauptverdienst am Gelingen des Bandes gebührt den Bearbeitern, Herrn Dr. Matthias Jaroch und Frau Dr. Mechthild Lindemann, zusammen mit der Wissenschaftlichen Leiterin, Frau Dr. Ilse Dorothee Pautsch. Ihnen sei für die erbrachte Leistung nachdrücklichst gedankt.

Ebenso haben Herr Lutz Holländer M.A. durch die Erstellung des Personenregisters, Herr Dr. Daniel Kosthorst durch Vorarbeiten für die Dokumentensammlung und Herr Dr. Wolfgang Hölscher durch die Beratung bei der Herstellung des Umbruchs wesentlich zur pünktlichen Fertigstellung der Edition beigetragen.

Berlin, den 1. August 2001

Hans-Peter Schwarz 


\section{Vorbemerkungen zur Edition}

Die „Akten zur Auswärtigen Politik der Bundesrepublik Deutschland 1953“ (Kurztitel: AAPD 1953) umfassen 383 Dokumente. Den abgedruckten Dokumenten gehen neben Vorwort und Vorbemerkungen ein Dokumentenverzeichnis, ein Literaturverzeichnis sowie ein Abkürzungsverzeichnis voran. Am Ende des Bandes findet sich ein Personen- und ein Sachregister sowie ein Organisationsplan des Auswärtigen Amts vom Mai/Juni 1953.

\section{Dokumentenauswahl}

Grundlage für die Fondsedition der „Akten zur Auswärtigen Politik der Bundesrepublik Deutschland 1953“ sind die Bestände des Politischen Archivs des Auswärtigen Amts (PA/AA). Besonderes Gewicht wurde auf den Bestand der Politischen Abteilung gelegt. Angemessene Berücksichtigung fanden aber auch die anderen Abteilungen des Auswärtigen Amts, insbesondere die Länder-, die Rechts- und die Kulturabteilung. Über die Bemühungen um eine Ratifizierung des Vertrags zur Gründung einer Europäischen Verteidigungsgemeinschaft gaben die Akten der „EVG-Delegation“ Aufschluß. Die Handakten des Leiters der Politischen Abteilung im Auswärtigen Amt, Herbert Blankenhorn, befinden sich zu einem Teil im PA/AA und zu einem anderen Teil im Bundesarchiv in Koblenz. Sie wurden ebenso ausgewertet wie einzelne Bestände aus der Stiftung Bundeskanzler-Adenauer-Haus in Rhöndorf (Nachlaß Adenauer).

Entsprechend ihrer Herkunft belegen die edierten Dokumente in erster Linie die außenpolitischen Aktivitäten des Bundeskanzlers und Bundesministers des Auswärtigen. Die Rolle anderer Akteure, insbesondere im parlamentarischen und parteipolitischen Bereich, wird beispielhaft dokumentiert, sofern eine Wechselbeziehung zum Auswärtigen Amt gegeben war.

Mit den ausgewählten Dokumenten werden erstmals Schriftstücke veröffentlicht, die bisher als Verschlußsachen der Geheimhaltung unterworfen waren. Dank einer entsprechenden Ermächtigung wurden den Bearbeitern die VSBestände des PA/AA ohne Einschränkung zugänglich gemacht und Anträge auf Herabstufung und Offenlegung von Schriftstücken beim Auswärtigen Amt ermöglicht. Kopien der offengelegten Schriftstücke, deren Zahl diejenige der in den AAPD 1953 edierten Dokumente weit übersteigt, werden im PA/AA zugänglich gemacht (Bestand B 150).

Nur eine äußerst geringe Zahl der für die Edition vorgesehenen Aktenstücke wurde nicht zur Veröffentlichung freigegeben. Hierbei handelt es sich vor allem um Dokumente, in denen personenbezogene Vorgänge im Vordergrund stehen oder die auch heute noch sicherheitsrelevante Angaben enthalten. Von einer Deklassifizierung ausgenommen war Schriftgut ausländischer Herkunft bzw. aus dem Bereich multilateraler oder internationaler Organisationen. Unberücksichtigt blieb ebenfalls nachrichtendienstliches Material.

Zur Dokumentation wesentlicher historischer Vorgänge wurden in Ausnahmefällen bereits an anderer Stelle veröffentlichte Schriftstücke von besonderer Bedeutung in die Auswahl aufgenommen. 


\section{Dokumentenfolge}

Die 383 edierten Dokumente sind in chronologischer Folge geordnet und mit laufenden Nummern versehen. Bei differierenden Datumsangaben auf einem Schriftstück, z.B. im Falle abweichender maschinenschriftlicher und handschriftlicher Datierung, ist in der Regel das früheste Datum maßgebend. Mehrere Dokumente mit demselben Datum sind, soweit möglich, nach der Uhrzeit eingeordnet. Erfolgt eine Datierung lediglich aufgrund sekundärer Hinweise (z.B. aus Begleitschreiben, beigefügten Vermerken usw.), wird dies in einer Anmerkung ausgewiesen. Bei Aufzeichnungen über Gespräche ist das Datum des dokumentierten Vorgangs ausschlaggebend, nicht der meist spätere Zeitpunkt der Niederschrift.

\section{Dokumentenkopf}

Jedes Dokument beginnt mit einem halbfett gedruckten Dokumentenkopf, in dem wesentliche formale Angaben zusammengefaßt werden. Auf Dokumentennummer und Dokumentenüberschrift folgen in kleinerer Drucktype ergänzende Angaben, so rechts außen das Datum. Links außen wird, sofern vorhanden, das Geschäftszeichen des edierten Schriftstücks einschließlich des Geheimhaltungsgrads (zum Zeitpunkt der Entstehung) wiedergegeben. Das Geschäftszeichen, das Rückschlüsse auf den Geschäftsgang zuläßt und die Ermittlung zugehörigen Aktenmaterials ermöglicht, besteht in der Regel aus der Kurzbezeichnung der ausfertigenden Arbeitseinheit sowie aus weiteren Elementen wie dem inhaltlich definierten Aktenzeichen, der Tagebuchnummer einschließlich verkürzter Jahresangabe und gegebenenfalls dem Geheimhaltungsgrad. Dokumentennummer, verkürzte Überschrift und Datum finden sich auch im Kolumnentitel über dem Dokument.

Den Angaben im Dokumentenkopf läßt sich die Art des jeweiligen Dokuments entnehmen. Aufzeichnungen sind eine in der Edition besonders häufig vertretende Dokumentengruppe. Der Verfasser wird jeweils in der Überschrift benannt, auch dann, wenn er sich nur indirekt erschließen läßt. Letzteres wird in einer Anmerkung vermerkt. Läßt sich ein solcher weder mittelbar noch unmittelbar nachweisen, wird die ausfertigende Arbeitseinheit (Abteilung, Referat oder Delegation) angegeben.

Breiten Raum nehmen Gesprächsaufzeichnungen sowie Niederschriften über Besprechungen und Konferenzen ein. Sie werden als solche in der Überschrift gekennzeichnet und chronologisch nach dem Gesprächs-, Besprechungs- oder Konferenzdatum eingeordnet, während Verfasser und Datum der Niederschrift - sofern ermittelbar - in einer Anmerkung ausgewiesen sind.

Eine wichtige Dokumentengruppe sind darüber hinaus Schreiben, erkennbar jeweils an der Nennung von Absender und Empfänger. Dazu gehören insbesondere die Schreiben der Bundesregierung, vertreten durch den Bundeskanzler und Bundesminister des Auswärtigen, an ausländische Regierungen, desgleichen auch Korrespondenz des Auswärtigen Amts mit anderen Ressorts oder mit Bundestagsabgeordneten. 
Eine weitere Gruppe von Dokumenten bildet der Schriftverkehr zwischen der Zentrale in Bonn und den Auslandsvertretungen. Diese erhielten ihre Informationen und Weisungen in der Regel mittels Drahterlaß, der fernschriftlich oder per Funk übermittelt wurde. Auch bei dieser Dokumentengruppe wird in der Überschrift der Verfasser genannt, ein Empfänger dagegen nur, wenn der Drahterlaß an eine einzelne Auslandsvertretung bzw. deren Leiter gerichtet war. Anderenfalls werden die Adressaten in einer Anmerkung aufgeführt. Bei Runderlassen an sehr viele oder an alle diplomatischen Vertretungen wird der Empfängerkreis nicht näher spezifiziert, um die Anmerkungen nicht zu überfrachten. Ebenso sind diejenigen Auslandsvertretungen nicht eigens aufgeführt, die nur nachrichtlich von einem Erlaß in Kenntnis gesetzt wurden. Ergänzend zum Geschäftszeichen wird im unteren Teil des Dokumentenkopfes links die Nummer des Drahterlasses sowie der Grad der Dringlichkeit angegeben. Rechts davon befindet sich das Datum und - sofern zu ermitteln - die Uhrzeit der Aufgabe. Ein Ausstellungsdatum wird nur dann angegeben, wenn es vom Datum der Aufgabe abweicht.

Der Dokumentenkopf bei einem im Auswärtigen Amt eingehenden Drahtbericht ist in Analogie zum Drahterlaß gestaltet. Außer Datum und Uhrzeit der Aufgabe wird auch der Zeitpunkt der Ankunft festgehalten, jeweils in Ortszeit.

In weniger dringenden Fällen verzichteten die Botschaften auf eine fernschriftliche Übermittlung und zogen die Form des mit Kurier übermittelten Schriftberichts vor. Beim Abdruck solcher Stücke werden im Dokumentenkopf neben der Überschrift mit Absender und Empfänger das Geschäftszeichen und das Datum genannt.

Die wenigen Dokumente, die sich keiner der beschriebenen Gruppen zuordnen lassen, sind aufgrund individueller Überschriften zu identifizieren.

Die Überschrift bei allen Dokumenten enthält die notwendigen Angaben zum Ausstellungs-, Absende- oder Empfangsort bzw. zum Ort des Gesprächs. Erfolgt keine besondere Ortsangabe, ist stillschweigend Bonn zu ergänzen. Hält sich der Verfasser oder Absender eines Dokuments nicht an seinem Dienstort auf, wird der Ortsangabe ein „z.Z." vorangesetzt.

Bei den edierten Schriftstücken handelt es sich in der Regel jeweils um die erste Ausfertigung oder - wie etwa bei den Drahtberichten - um eines von mehreren gleichrangig nebeneinander zirkulierenden Exemplaren. Statt einer Erstausfertigung mußten gelegentlich ein Durchdruck, eine Abschrift, eine Ablichtung oder ein vervielfältigtes Exemplar (Matrizenabzug) herangezogen werden. Ein entsprechender Hinweis findet sich in einer Anmerkung. In wenigen Fällen sind Entwürfe abgedruckt und entsprechend in den Überschriften kenntlich gemacht.

\section{Dokumententext}

Unterhalb des Dokumentenkopfes folgt - in normaler Drucktype - der Text des jeweiligen Dokuments, einschließlich des Betreffs, der Anrede und der Unterschrift. Die Dokumente werden ungekürzt veröffentlicht. Sofern in Ausnahmefällen Auslassungen vorgenommen werden müssen, ist dies kenntlich gemacht 
und in einer Anmerkung erläutert. Textergänzungen der Bearbeiter stehen in eckigen Klammern.

Offensichtliche Schreib- und Interpunktionsfehler werden stillschweigend korrigiert. Eigentümliche Schreibweisen bleiben nach Möglichkeit erhalten; im Bedarfsfall wird jedoch vereinheitlicht bzw. modernisiert. Dies trifft teilweise auch auf fremdsprachige Orts- und Personennamen zu, deren Schreibweise nach den im Auswärtigen Amt gebräuchlichen Regeln wiedergegeben wird.

Selten vorkommende und ungebräuchliche Abkürzungen werden in einer Anmerkung aufgelöst. Typische Abkürzungen von Institutionen, Parteien etc. werden allerdings übernommen. Hervorhebungen in der Textvorlage, also etwa maschinenschriftliche Unterstreichungen oder Sperrungen, werden nicht wiedergegeben. Der Kursivdruck dient dazu, bei Gesprächsaufzeichnungen die Sprecher voneinander abzuheben. Im äußeren Aufbau (Absätze, Überschriften usw.) folgt das Druckbild nach Möglichkeit der Textvorlage.

Unterschriftsformeln werden vollständig wiedergegeben. Ein handschriftlicher Namenszug ist nicht besonders gekennzeichnet, eine Paraphe mit Unterschriftscharakter wird aufgelöst (mit Nachweis in einer Anmerkung). Findet sich auf einem Schriftstück der Name zusätzlich maschinenschriftlich vermerkt, bleibt dies unerwähnt. Ein maschinenschriftlicher Name, dem ein ,gez." vorangestellt ist, wird entsprechend übernommen; fehlt in der Textvorlage der Zusatz "gez.", wird er in eckigen Klammern ergänzt. Weicht das Datum der Paraphe vom Datum des Schriftstückes ab, wird dies in der Anmerkung ausgewiesen.

Unter dem Dokumententext wird die jeweilige Fundstelle des Schriftstückes in halbfetter Schrifttype nachgewiesen. Bei Dokumenten aus dem PA/AA wird auf die Angabe des Archivs verzichtet und nur der jeweilige Bestand mit Bandnummer genannt. Dokumente aus VS-Beständen sind mit der Angabe „VS-Bd.“ versehen. Bei Dokumenten anderer Herkunft werden Archiv und Bestandsbezeichnung angegeben. Liegt ausnahmsweise ein Schriftstück bereits veröffentlicht vor, so wird dies in einer gesonderten Anmerkung nach der Angabe der Fundstelle ausgewiesen.

\section{Kommentierung}

In Ergänzung zum Dokumentenkopf enthalten die Anmerkungen formale Hinweise und geben Auskunft über wesentliche Stationen im Geschäftsgang. Angaben technischer Art, wie Registraturvermerke oder standardisierte Verteiler, werden nur bei besonderer Bedeutung erfaßt. Wesentlich ist dagegen die Frage, welche Beachtung das jeweils edierte Dokument gefunden hat. Dies läßt sich an den Paraphen maßgeblicher Akteure sowie an den - überwiegend handschriftlichen - Weisungen, Bemerkungen oder auch Reaktionen in Form von Frage- oder Ausrufungszeichen ablesen, die auf dem Schriftstück selbst oder auf Begleitschreiben und Begleitvermerken zu finden sind. Die diesbezüglichen Merkmale sowie damit in Verbindung stehende Hervorhebungen (Unterstreichungen oder Anstreichungen am Rand) werden in Anmerkungen nachgewiesen. Auf den Nachweis sonstiger An- oder Unterstreichungen wird ver- 
zichtet. Abkürzungen in handschriftlichen Passagen werden in eckigen Klammern aufgelöst, sofern sie nicht im Abkürzungsverzeichnis aufgeführt sind.

In den im engeren Sinn textkritischen Anmerkungen werden nachträgliche Korrekturen oder textliche Änderungen des Verfassers und einzelner Adressaten festgehalten, sofern ein Konzipient das Schriftstück entworfen hat. Unwesentliche Textverbesserungen sind hiervon ausgenommen. Ferner wird auf einen systematischen Vergleich der Dokumente mit Entwürfen ebenso verzichtet wie auf den Nachweis der in der Praxis üblichen Einarbeitung von Textpassagen in eine spätere Aufzeichnung oder einen Drahterlaß.

Die Kommentierung soll den historischen Zusammenhang der edierten Dokumente in ihrer zeitlichen und inhaltlichen Abfolge sichtbar machen, weiteres Aktenmaterial und anderweitiges Schriftgut nachweisen, das unmittelbar oder mittelbar angesprochen wird, sowie Ereignisse oder Sachverhalte näher erläutern, die dem heutigen Wissens- und Erfahrungshorizont ferner liegen und aus dem Textzusammenhang heraus nicht oder nicht hinlänglich zu verstehen sind.

Besonderer Wert wird bei der Kommentierung darauf gelegt, die Dokumente durch Bezugsstücke aus den Akten der verschiedenen Arbeitseinheiten des Auswärtigen Amts bis hin zur Leitungsebene zu erläutern. Zitate oder inhaltliche Wiedergaben sollen die Entscheidungsprozesse erhellen und zum Verständnis der Dokumente beitragen. Dadurch wird zugleich Vorarbeit geleistet für eine vertiefende Erschließung der Bestände des PA/AA. Um die Identifizierung von Drahtberichten bzw. -erlassen zu erleichtern, werden außer dem Verfasser und dem Datum die Drahtberichtsnummer und, wo immer möglich, die Drahterlaßnummer angegeben.

Findet in einem Dokument veröffentlichtes Schriftgut Erwähnung - etwa Abkommen, Gesetze, Reden oder Presseberichte -, so wird die Fundstelle nach Möglichkeit genauer spezifiziert. Systematische Hinweise auf archivalische oder veröffentlichte Quellen, insbesondere auf weitere Bestände des PA/AA, erfolgen nicht. Sekundärliteratur wird generell nicht in die Kommentierung aufgenommen.

Angaben wie Dienstbezeichnung, Dienststellung, Funktion, Dienstbehörde und Nationalität dienen der eindeutigen Identifizierung der in der Kommentierung vorkommenden Personen. Bei Bundesministern erfolgt ein Hinweis zum jeweiligen Ressort nur im Personenregister. Eine im Dokumententext lediglich mit ihrer Funktion genannte Person wird nach Möglichkeit in einer Anmerkung namentlich nachgewiesen. Davon ausgenommen sind der jeweilige Bundespräsident, Bundeskanzler und Bundesminister des Auswärtigen.

Die Bezeichnung einzelner Staaten wird so gewählt, daß Verwechslungen ausgeschlossen sind. Als Kurzform für die Deutsche Demokratische Republik kommen in den Dokumenten die Begriffe SBZ oder DDR vor und werden so wiedergegeben. Der in der Forschung üblichen Praxis folgend, wird jedoch in der Kommentierung, den Verzeichnissen sowie den Registern der Begriff DDR verwendet. Das Adjektiv „deutsch" findet nur bei gesamtdeutschen Belangen oder dann Verwendung, wenn eine eindeutige Zuordnung gegeben ist. Der westliche Teil von Berlin wird als Berlin (West), der östliche Teil der Stadt als Ost-Berlin bezeichnet. 
Für Publikationen wie Editionen, Geschichtskalender und Memoiren werden Kurztitel oder Kurzformen eingeführt, die sich über ein entsprechendes Verzeichnis auflösen lassen. Der Platzersparnis dienen ebenfalls die Rückverweise auf bereits an anderer Stelle ausgeführte Anmerkungen.

Wie bei der Wiedergabe der Dokumente finden auch in den Anmerkungen die im Auswärtigen Amt gebräuchlichen Regeln für die Transkription fremdsprachlicher Namen und Begriffe Anwendung. Bei Literaturangaben in russischer Sprache wird die im wissenschaftlichen Bereich übliche Transliterierung durchgeführt.

\section{Verzeichnisse}

Das Dokumentenverzeichnis ist chronologisch angelegt. Es bietet zu jedem Dokument folgende Angaben: Die halbfett gedruckte Dokumentennummer, Datum und Überschrift, die Fundseite sowie eine inhaltliche Kurzübersicht.

Das Literaturverzeichnis enthält die Publikationen, die zur Kommentierung herangezogen und mit Kurztiteln oder Kurzformen versehen wurden. Diese sind alphabetisch geordnet und werden durch bibliographische Angaben aufgelöst.

Das Abkürzungsverzeichnis führt die im Dokumententeil vorkommenden Abkürzungen auf, insbesondere von Firmen, Organisationen, Parteien und Dienstbezeichnungen sowie sonstige im diplomatischen Schriftverkehr übliche Abbreviaturen. Nicht aufgenommen werden geläufige Abkürzungen wie „z.B.“, „d.h.“, „m.E.“, „u. U.“ und „usw." sowie Abkürzungen, die im Dokumententext oder in einer Anmerkung erläutert sind.

\section{Register und Organisationsplan}

Im Personenregister werden in der Edition vorkommende Personen unter Nennung derjenigen politischen, dienstlichen oder beruflichen Funktionen aufgeführt, die im inhaltlichen Zusammenhang der Dokumente wesentlich sind. Das Sachregister ermöglicht einen thematisch differenzierten Zugriff auf die einzelnen Dokumente. Näheres ist den Hinweisen zur Benutzung des jeweiligen Registers auf S. 1160 und S. 1206 zu entnehmen.

Der Organisationsplan vom Mai/Juni 1953 zeigt die Struktur des Auswärtigen Amts und informiert über die Namen der Leiter der jeweiligen Arbeitseinheiten. 

Verzeichnisse 



\section{Dokumentenverzeichnis}

Abs resümiert die Besprechungen vom 16. September bis 9. Dezember 1952 in London zu den Abkommen über die deutschen Nachkriegsschulden.

Pawelke berichtet über Gespräche mit Ministerpräsident Naguib und dem ägyptischen Außenminister Fawzi. Thema war die Beilegung des Konflikts mit den arabischen Staaten, der durch das Abkommen mit Israel entstanden war.

Salat erörtert die finanzielle Beteiligung der Bundesrepublik an Projekten des Europäischen Rats für kernphysikalische Forschung (CERN).

Brentano informiert über ein Gespräch mit einem Vertreter des Bischofs von Trier. Im Mittelpunkt stand die von Frankreich gewünschte Ernennung eines apostolischen Administrators in Saarbrücken.

Kordt faßt ein Gespräch mit dem Leiter der finnischen Handelsvertretung, Munkki, über die Errichtung von Handelsvertretungen der DDR und der Bundesrepublik in Helsinki zusammen. Staatssekretär Hallstein

Riesser berichtet über ein Gespräch mit dem amerikanischen Bankier Baruch. Thema war die europäische Verteidigung.

Hallstein übermittelt eine Stellungnahme des Beauftragten des Bundeskanzlers, Blank, zur französischen Forderung nach Zusatzprotokollen zum EVG-Vertrag.

Allardt informiert über die Beteiligung von Firmen aus der Bundesrepublik an den Vorarbeiten für die Errichtung eines Staudamms bei Assuan. 
9 08.01. Aufzeichnung des Staatssekretärs Hallstein

S. 36

Gegenstand des Vermerks für Bundeskanzler Adenauer sind die Handelsbesprechungen mit Frankreich.

Oncken äußert sich zur Behandlung der deutschen Frage in der Öffentlichkeit. an das Auswärtige Amt

Holleben berichtet über ein Gespräch mit dem niederländischen Außenminister Beyen. Themen waren die politische und wirtschaftliche Integration Europas sowie der EVG-Vertrag.

Allardt resümiert ein Gespräch des Staatssekretärs Hallstein mit dem Berater der ägyptischen Regierung, Voss, über die Beilegung des Konflikts mit den arabischen Staaten.

Amt

Nöldeke informiert über Pläne zur Vergrößerung des dänischen Truppenkontingents in Schleswig-Holstein. Amt

Riesser berichtet über ein Gepräch mit dem ehemaligen amerikanischen Hohen Kommissar McCloy, der eine Stellungnahme des amerikanischen Außenministers Acheson zur Reaktion des Bundeskanzlers Adenauer auf den französischen Wunsch nach Zusatzprotokollen zum EVG-Vertrag übermittelte. Staatssekretär Hallstein

Krekeler informiert über ein Gespräch des Generalkonsuls Riesser mit dem ehemaligen amerikanischen Hohen Kommissar McCloy. Themen waren die französische Haltung zum EVGVertrag, die Ernennung eines neuen amerikanischen Hohen Kommissars für Deutschland sowie die Vorstellungen des amerikanischen Bankiers Baruch zur europäischen Verteidigung.

Thierfelder nimmt Stellung zum Schreiben des französischen Außenministers Schuman vom 21. Dezember 1952 an Bundeskanzler Adenauer zur Saarfrage. 

von Etzdorf

Etzdorf faßt eine Unterredung des Staatssekretärs Hallstein mit dem jugoslawischen Botschafter Iveković über die bilateralen Beziehungen zusammen. Auswärtige Amt

Krekeler übermittelt eine Einschätzung zur Europa-Politik des zukünftigen Präsidenten Eisenhower. Auswärtige Amt

Kessel berichtet über ein Gespräch mit dem französischen Delegationsleiter beim Interimsausschuß der EVG-Konferenz, Alphand. Im Mittelpunkt stand der französische Wunsch nach Zusatzprotokollen zum EVG-Vertrag.

Pawelke resümiert ein Gespräch mit dem ägyptischen AuBenminister Fawzi. Themen waren die Entsendung einer Wirtschaftsdelegation nach Kairo und die Sitzung des Politischen Komitees der Arabischen Liga vom Vortag. Auswärtige Amt

Kessel berichtet von einem Gespräch mit dem belgischen Ständigen Vertreter bei der NATO, de Staercke, über die französischen Haltung zur EVG.

Kordt informiert über ein Gespräch mit dem belgischen Botschafter Muûls zu Grenzfragen.

Jansen übermittelt Äußerungen des luxemburgischen Außenministers Bech zur Tagung der Ad-hoc-Versammlung für die Gründung einer Europäischen Politischen Gemeinschaft vom 7. bis 10. Januar 1953 in Straßburg.

\section{z.Z. Bad Wörishofen}

Hallstein bittet Hermes, die Leitung der Delegation der Bundesrepublik bei den Konferenzen über die Organisation der Europäischen Agrarmärkte zu übernehmen. 

Auswärtige Amt

Kessel berichtet von einem Gespräch des Generalleutnants a.D. Speidel mit dem stellvertretenden italienischen Delegationsleiter im Interimsausschuß der EVG-Konferenz, Malfatti, über die französische Haltung zur EVG. Auswärtige Amt

Kessel informiert über eine Unterredung mit dem Stellvertreter des amerikanischen Vertreters bei der EGKS, Tomlinson. Im Mittelpunkt stand der französische Wunsch nach Zusatzprotokollen zum EVG-Vertrag.

Pawelke erläutert die Gründe für die Weigerung Ägyptens, sich auf der Sitzung des Politischen Komitees der Arabischen Liga vom 14. Januar 1953 für die Aufhebung des Boykottbeschlusses gegen die Bundesrepublik einzusetzen.

Kielmansegg resümiert eine Ressortbesprechung über die Haltung der Bundesregierung gegenüber dem französischen Wunsch nach Zusatzprotokollen zum EVG-Vertrag. und des Gesandten I. Klasse Ophüls

Mögliche französische Forderungen nach Zusatzprotokollen zum EVG-Vertrag werden analysiert und bewertet. Blank

Blank informiert über ein Gespräch mit dem französischen Stellvertretenden Hohen Kommissar Bérard zum Stand der Ratifizierung des EVG-Vertrags und zur Bedeutung von Zusatzprotokollen. Auswärtige Amt

Rosen berichtet über die wachsende Skepsis gegenüber der Bundesrepublik in der britischen Öfentlichkeit.

Hausenstein faßt ein Gespräch mit dem ehemaligen französischen Außenminister Schuman zusammen. Erörtert wurden dessen Rücktritt, die Saarfrage, die Haltung gegenüber der 
UdSSR, das deutsch-französische Verhältnis und die europäische Verteidigung.

Pawelke informiert über die Sitzung des Politischen Komitees der Arabischen Liga vom 14. Januar 1953. Ferner äußert er sich zum Zeitpunkt einer Ratifizierung des Abkommens mit Israel durch Bundesrat und Bundestag.

Salat berichtet über die Haltung des Vatikans zur Verzögerung bei der Besetzung des Botschafterpostens beim Heiligen Stuhl und zu den Plänen, eine Gesandtschaft Bayerns beim Heiligen Stuhl zu errichten.

Kordt befaßt sich mit der Aufnahme diplomatischer Beziehungen zu Österreich.

Frowein erörtert Möglichkeiten zur Vorfinanzierung der im Abkommen vom 10. September 1952 vorgesehenen Öllieferungen an Israel.

Im Mittelpunkt stehen die Saarfrage und die französische Forderung nach Zusatzprotokollen zum EVG-Vertrag.

Ophüls resümiert ein Gespräch mit dem Stellvertreter des amerikanischen Vertreters bei der EGKS, Tomlinson. Thema war die französische Forderung nach Zusatzprotokollen zum EVGVertrag. Overbeck

Overbeck notiert den Sachstand in der Frage der Unterzeichnung von vier Konventionsentwürfen des Europarats durch das Saargebiet.

Hallstein gibt einen Überblick zum Stand der Saarfrage. 
41

29.01. Staatssekretär Hallstein an Staatssekretär Westrick, Bundesministerium für Wirtschaft

Hallstein erteilt Instruktionen für die Wirtschaftsgespräche mit den arabischen Staaten.

\section{z.Z. London}

Weiz berichtet über die erste Sitzung des Drei-Mächte-Ausschusses mit der Delegation für Auslandsschulden und den Vertretern der an der Konferenz über deutsche Auslandsschulden teilnehmenden Staaten. Thema war der Artikel 5 des Entwurfs für ein Abkommen über deutsche Auslandsschulden.

Böker resümiert ein Gespräch mit dem indonesischen Gesandten Zain über eine Entsendung von Militärberatern aus der Bundesrepublik nach Indonesien.

Hallstein berichtet über einen Aufenthalt in London. Im Mittelpunkt der Gespräche standen die europäische Integration, die Ratifizierung des Abkommens vom 10. September 1952 mit Israel, die internationale Gesamtlage, die Entflechtung der KruppBetriebe sowie Währungsfragen.

Biermann befaßt sich mit der Frage, ob bzw. wann die Bundesrepublik einen Antrag auf Aufnahme in die UNO stellen sollte.

Granow resümiert ein Gespräch mit dem schwedischen Delegierten bei den Besprechungen über deutsche Auslandsschulden, Brück, zu Fragen der Schuldenverrechnung mit Schweden.

Adenauer nimmt Stellung zur Saarfrage. amerikanischen Außenminister Dulles

Im Mittelpunkt stehen die Ratifizierung des Generalvertrags und des EVG-Vertrags sowie des Abkommens vom 10. September 1952 mit Israel, die Saarfrage sowie das Flüchtlings- und Schuldenproblem. 
Erörtert wird die Stellungnahme der Bundesregierung zu niederländischen Vorschlägen für eine europäische wirtschaftliche Integration. Ministerialdirektor Blankenhorn

Allardt informiert über den Beginn der Wirtschaftsbesprechungen mit Ägypten und weiteren arabischen Staaten in Kairo.

05.02. Botschafter Terdenge, Buenos Aires, an das Auswärtige

Amt

Terdenge berichtet über die Rückgabe deutscher Warenzeichen und Patente.

Sattler unterrichtet über ein Gespräch mit dem Vorsitzenden der Interalliierten Sequesterkommission, McIvor. Thema war die Zukunft der deutschen Kulturinstitute in Italien.

Hallstein faßt eine Unterredung mit dem französischen Hohen Kommissar François-Poncet zusammen. Im Mittelpunkt standen die Saarfrage sowie Äußerungen des Bundeskanzlers Adenauer auf der Pressekonferenz am 6. Februar 1953. Auswärtige Amt

Kessel berichtet von einem Gespräch mit dem belgischen Ständigen Vertreter bei der NATO, de Staercke, über den Besuch des amerikanischen Außenministers Dulles in Brüssel. Auswärtige Amt

Kessel resümiert ein Gespräch mit dem belgischen Ständigen Vertreter bei der NATO, de Staercke. Gegenstand waren Äußerungen des amerikanischen Außenministers Dulles bei dessen Besuch in Bonn zur Oder-Neiße-Linie. Botschafter Pawelke, Kairo

Hallstein erteilt Weisung, wie auf den angekündigten Besuch einer Wirtschaftsdelegation aus der DDR in Kairo zu reagieren sei. 

Wirtschaft, z.Z. Kairo, an Staatssekretär Hallstein

Westrick informiert über den Verlauf der Wirtschaftsbesprechungen mit Ägypten und weiteren arabischen Staaten.

Adenauer bittet um die Bereitstellung von Mitteln zur Vorfinanzierung der im Abkommen vom 10. September 1952 vorgesehenen Öllieferungen an Israel. Amt

Von der Esch berichtet über ein Gespräch mit dem syrischen AuBenminister Rifai. Themen waren die internationale Lage sowie die Beilegung des Konflikts mit den arabischen Staaten durch Einschaltung der UNO.

Im Mittelpunkt stehen die wirtschaftlichen Aspekte der Saarfrage.

Stoecker vermerkt, daß der französische Delegationsleiter beim Interimsausschu $\beta$ der EVG-Konferenz, Alphand, den anderen Delegationsleitern die Vorschläge für Zusatzprotokolle zum EVG-Vertrag überreicht habe.

Kroll informiert über ein Gespräch mit Staatspräsident Tito anläßlich der Übergabe seines Beglaubigungsschreibens. Themen waren die bilateralen Wirtschaftsbeziehungen und die Entlassung von deutschen Kriegsgefangenen.

Die Bundesregierung legt ihre Haltung zu den französischen Vorschlägen für Zusatzprotokolle zum EVG-Vertrag dar. Auswärtige Amt

Kessel berichtet über ein Gespräch mit dem Stellvertreter des amerikanischen Vertreters bei der EGKS, Tomlinson. Gegenstand waren die französischen Vorschläge für Zusatzprotokolle zum EVG-Vertrag. 
65 16.02. Gesandtschaftsrat Federer, Washington, an das

S. 191 Auswärtige Amt

Federer informiert über ein Gespräch mit dem Abteilungsleiter im amerikanischen Außenministerium, Riddleberger. Erörtert wurden die französischen Vorschläge für Zusatzprotokolle zum EVG-Vertrag. Außenminister Bidault

Adenauer nimmt Stellung zur Beteiligung des Saargebiets an einer Europäischen Politischen Gemeinschaft. von Falkenstein

Trützschler äußert sich zur Aufnahme von Verhandlungen mit Israel über das Recht deutscher Schiffe, in Durchführung des Abkommens vom 10. September 1952 israelische Häfen anzulaufen. Auswärtige Amt

Bayern befaßt sich mit der Unterzeichnung eines Kulturabkommens mit Spanien in Madrid. Auswärtige Amt

Federer informiert über die amerikanische Haltung zu einer Verstärkung des Bundesgrenzschutzes.

Hallstein resümiert eine Besprechung mit Bundeskanzler Adenauer und dem Beauftragten des Bundeskanzlers, Blank, in der eine Weisung zu den französischen Vorschlägen für Zusatzprotokolle zum EVG-Vertrag formuliert wurde. Amt

Walther berichtet über ein Gespräch mit dem Abteilungsleiter im französischen Außenministerium, Seydoux. Themen waren die britisch-französischen Regierungsbesprechungen in London über eine britische Assoziierung mit der EVG und die französischen Vorschläge für Zusatzprotokolle zum EVG-Vertrag.

Bassler bewertet eine Rede des Vorsitzenden des Außenpolitischen Ausschusses des dänischen Parlaments, Hedtoft, zur Po- 
litik gegenüber der dänischen Minderheit in Schleswig-Holstein.

73

20.02. Bundeskanzler Adenauer an den Geschäftsführenden Vorsitzenden der Alliierten Hohen Kommission, Conant

Adenauer nimmt zu den Bedenken Stellung, die von den Drei Mächten gegen eine geplante Reform der Einkommen- und Körperschaftssteuer geäußert wurden.

$\mathbf{7 4}$

20.02. Aufzeichnung des Vortragenden Legationsrats Allardt

Allardt erläutert die Gründe für die arabische Kritik am Abkommen vom 10. September 1952 mit Israel.

20.02. Aufzeichnung des Legationsrats I. Klasse Trützschler von Falkenstein

Trützschler erörtert das weitere Vorgehen im Falle der im Oradour-Prozeß verurteilten Deutschen nach der Amnestierung der verurteilten Elsässer durch die französische Regierung.

20.02. Aufzeichnung des Vortragenden Legationsrats Allardt

Allardt bewertet die Wirtschaftsverhandlungen mit Ägypten und weiteren arabischen Staaten.

21.02. Bundeskanzler Adenauer an den Geschäftsführenden Vorsitzenden der Alliierten Hohen Kommission, Conant

Adenauer übermittelt ein Memorandum mit der Bitte um Verbesserung der Bewaffnung des Bundesgrenzschutzes und der Bereitschaftspolizei der Länder.

21.02. Gesandtschaftsrat Federer, Washington, an das Auswärtige Amt

Federer berichtet über ein Gespräch mit dem Abteilungsleiter im amerikanischen Außenministerium, Riddleberger. Thema war die amerikanische Haltung gegenüber den französischen Vorschlägen für Zusatzprotokolle zum EVG-Vertrag.

Hallstein erklärt die Bereitschaft der Bundesregierung, eine Sachverständigenkommission zur Prüfung des Assuan-Staudamm-Projekts nach Ägypten zu entsenden.

\subsection{Aufzeichnung des Vortragenden Legationsrats Sachs}

Sachs resümiert die in der Sitzung der Außenministerkonferenz der EGKS-Mitgliedstaaten in Rom erörterten niederländischen Vorschläge zur europäischen wirtschaftlichen Integration.

S. 207

S. 216 
81 24.02. Außenministerkonferenz der EGKS-Mitgliedstaaten in

S. 232 Rom

Die französischen Vorschläge für Zusatzprotokolle zum EVGVertrag werden erörtert. Rom

Themen sind die französischen Vorschläge für Zusatzprotokolle zum EVG-Vertrag und die Ratifizierung des Vertragswerks in den Unterzeichnerstaaten.

Mosler resümiert eine Besprechung mit dem amerikanischen Stellvertretenden Hohen Kommissar Harris. Gegenstand waren die Gespräche der Bundesregierung mit Drittstaaten über das dortige deutsche Vermögen.

84 26.02. Gespräch des Bundeskanzlers Adenauer mit dem französischen Außenminister Bidault in Rom

Thema ist die Saarfrage. Kessel, Paris

Kessel gibt ein Gespräch mit Pater Leiber wieder. Themen waren das Befinden von Papst Pius XII. und der Einfluß der Bundesrepublik beim Heiligen Stuhl, die Situation in der UdSSR sowie die kirchenpolitische Lage in Osteuropa.

Nöhring nimmt zu jemenitischen Kreditwünschen Stellung.

\section{Adenauer}

Vor der Reise von Adenauer in die USA unterbreitet Gerstenmaier Überlegungen für eine engere Zusammenarbeit mit den USA.

Klee unterrichtet über das Zögern der honduranischen Regierung bei der Aufhebung des Kriegszustandes mit Deutschland und die ungeklärten Eigentumsverhältnisse von Deutschen in Honduras. 
89

03.03. Generalkonsul Hausenstein, Paris, an das Auswärtige

Amt

Hausenstein berichtet über die Verhandlungen zwischen Frankreich und dem Saargebiet bezüglich einer Revision der Saarkonventionen.

05.03. Botschafter von Hentig, Djakarta, an das Auswärtige Amt

Hentig faßt ein Gespräch mit Präsident Sukarno zusammen. Thema war die Entsendung von Militärexperten aus der Bundesrepublik nach Indonesien.

91 05.03. Vortragender Legationsrat von Kessel, Paris, an das Auswärtige Amt

Kessel resümiert die Verhandlungen über die von Frankreich vorgelegten Zusatzprotokolle zum EVG-Vertrag.

06.03. Aufzeichnung des Vortragenden Legationsrats von Haeften

Haeften erörtert die Beteiligung des Saargebiets an den Konventionen des Europarats.

Kordt unterrichtet über Maßnahmen zur Beilegung des Konflikts mit den arabischen Staaten. französischen Außenminister Bidault

Themen sind die Saarfrage, die friedliche Wiedervereinigung Deutschlands unter Einschluß der Gebiete östlich der OderNeiße-Linie und die von Frankreich vorgelegten Zusatzprotokolle zum EVG-Vertrag. Bundesministerium für Wirtschaft

Heipertz informiert über den West-Ost-Handel.

Blankenhorn erinnert an Erklärungen von seiten der Bundesregierung zur Wiedervereinigung mit ausschließlich friedlichen Mitteln. 
der EVG-Konferenz, Bruce, zusammen, in dem die Ratifizierung des EVG-Vertrags in den einzelnen Staaten thematisiert wurde.

Kroll informiert über die jugoslawische Einschätzung der sowjetischen Politik nach dem Tod des Vorsitzenden des Ministerrats und Generalsekretärs des ZK der KPdSU, Stalin.

Hallstein gibt ein Gespräch des Bundeskanzlers Adenauer mit dem französischen Hohen Kommissar François-Poncet wieder. Im Mittelpunkt stand die Moselkanalisierung.

13.03. Vortragender Legationsrat von Kessel, Paris, an das Auswärtige Amt

Kessel berichtet über italienische Sondierungen in Washington wegen angeblicher französischer Wünsche nach Bildung einer politischen "standing group".

101 13.03. Vortragender Legationsrat von Kessel, Paris, an das Auswärtige Amt

Kessel übermittelt Äußerungen von SHAPE zur Berücksichtigung der Bundesrepublik bei der Vergabe von militärischen Führungspositionen in der EVG und zu den Besprechungen in Bonn über Dislozierung. Auswärtige Amt

Krekeler informiert über amerikanische Besorgnisse, daß die Bundesrepublik separate Absprachen mit der UdSSR über die Wiedervereinigung Deutschlands treffen könnte. Trützschler von Falkenstein

Trützschler äußert sich zur Einstellung von Fahndungsmaßnahmen gegen die wegen Kriegsverbrechen verurteilten Deutschen Morio und Seuffert, die aus norwegischer Haft geflohen sind.

Die weiteren Verhandlungen mit Frankreich über die Saarfrage werden erörtert. Auswärtige Amt

Krekeler berichtet über ein Gespräch mit Präsident Eisenhower zur Politik der USA gegenüber der UdSSR. 
Bayern resümiert ein Gespräch mit dem spanischen Außenminister Martín Artajo. Erörtert wurden die Verhandlungen Spaniens mit den USA über ein Verteidigungsabkommen, die bilateralen Beziehungen und die spanische Beteiligung an der Verteidigung Europas.

Hermes berichtet über die Besprechungen der Landwirtschaftsminister der EGKS-Staaten und die Konferenz für die Organisation der Europäischen Agrarmärkte in Paris. Auswärtige Amt

Krekeler informiert über Gespräche mit dem amerikanischen Außenminister Dulles und Staatssekretär Smith. Im Mittelpunkt standen die Ratifizierung des EVG-Vertrags und die Politik der USA gegenüber der UdSSR.

\section{27.03. Aufzeichnung des Auswärtigen Amts}

Über die Verhandlungen im Juristenausschuß zu den von Frankreich vorgelegten Zusatzprotokollen zum EVG-Vertrag wird berichtet. Bundeskanzler Adenauer und Staatssekretär Hallstein

Blankenhorn berichtet von einem Gespräch mit dem Abteilungsleiter im amerikanischen Außenministerium, Riddleberger, über die amerikanisch-französischen Regierungsbesprechungen und die Vorbereitung der Reise von Adenauer in die USA. Bundeskanzler Adenauer und Staatssekretär Hallstein

Blankenhorn unterrichtet über Gespräche mit dem Berater im amerikanischen Außenministerium, MacArthur, und dem Abteilungsleiter im amerikanischen Außenministerium, Nash. Erörtert wurden die amerikanisch-französischen Regierungsbesprechungen und die Vorbereitung der Reise von Adenauer in die USA. Blankenhorn

Pawelke berichtet über ein Gespräch mit dem ägyptischen Oberst Nasser. Thema war die Beilegung des Konflikts zwischen den arabischen Staaten und Israel. 

Eisenhower in Washington

Im Mittelpunkt stehen die europäische Integration, die Saarfrage und die Politik der UdSSR.

114 07.04. Gespräch des Bundeskanzlers Adenauer mit dem amerikanischen Außenminister Dulles in Washington

Erörtert werden das Inkrafttreten des EVG-Vertrags, die Saarfrage, der Status der jeweiligen Auslandsvertretungen, die Politik der UdSSR, die Freilassung wegen Kriegsverbrechen verurteilter Deutscher, der finanzielle Verteidigungsbeitrag der Bundesrepublik und die Ausrüstung ihrer Streitkräfte durch die USA.

Themen sind die amerikanische Unterstützung bei der Eingliederung von Flüchtlingen, die Freigabe beschlagnahmter deutscher Vermögenswerte und Warenzeichen, das amerikanische "Off-shore"-Programm, der West-Ost-Handel, die Freilassung wegen Kriegsverbrechen verurteilter Deutscher und die Zivilverteidigung der Bundesrepublik.

Im Anschluß an einen Aufenthalt in Kairo informiert Munzel über die Reaktion der arabischen Staaten auf die Ratifizierung des Vertrags mit Israel durch die Bundesrepublik und den Beginn von Sachverständigenbesprechungen mit Ägypten über den Bau des Assuan-Staudamms.

Blankenhorn resümiert ein Gespräch mit dem britischen Hohen Kommissar Kirkpatrick, mit Bundesminister Dehler und mit dem Unterstaatssekretär im britischen Außenministerium, Roberts. Themen waren die Naumann-Affäre, die aktuellen Entwicklungen in der UdSSR und die europäische Einigung.

118 14.04. Generalkonsul I. Klasse Schlange-Schöningen, London, an das Auswärtige Amt

Schlange-Schöningen berichtet über den Konflikt zwischen Ägypten und Großbritannien um die Räumung der Suezkanalzone sowie die britischen Besorgnisse wegen der Tätigkeit deutscher Militärexperten für die ägyptische Regierung. 
119

15.04. Botschaftsrat von Walther, Paris, an Ministerialdirektor Blankenhorn

Walther weist auf die Auswirkungen hin, welche die Saarfrage und die Politik der UdSSR auf die französische Haltung zum EVG-Vertrag haben könnte.

120 15.04. Gesandtschaftsrat I. Klasse Federer, Washington, an das Auswärtige Amt

Federer berichtet über den Abschluß des Kulturabkommens mit den USA.

121

18.04. Gespräch des Bundeskanzlers Adenauer mit Ministerpräsident St. Laurent in Ottawa

Im Mittelpunkt stehen die Freilassung wegen Kriegsverbrechen verurteilter Deutscher, die Einwanderungsmöglichkeiten für Flüchtlinge aus der DDR nach Kanada, die Freigabe von beschlagnahmten deutschen Vermögen, die bilateralen Handelsbeziehungen sowie die Bedrohung Europas durch die UdSSR.

122

18.04. Gespräch des Bundeskanzlers Adenauer mit den kanadischen Ministern Abbott, Claxton und Harris in Ottawa

Themen sind die Einwanderungsmöglichkeiten für Flüchtlinge aus der DDR nach Kanada, die Freigabe von beschlagnahmten deutschen Vermögen und die Deutschlandpolitik der UdSSR.

123 20.04. Gesandter Katzenberger, Dublin, an das Auswärtige Amt

Katzenberger berichtet über die irische Forderung nach Entschädigung für die Verluste und Schäden aus dem Zweiten Weltkrieg.

124

23.04. Aufzeichnung des Gesandten I. Klasse Ophüls

Ophüls befaßt sich mit den wirtschaftlichen Bestimmungen im Entwurf für einen Vertrag über die Satzung der Europäischen Gemeinschaft.

125

24.04. Aufzeichnung des Vortragenden Legationsrats Overbeck

Overbeck diskutiert die Vorgehensweise bei der Inkraftsetzung der Konventionen des Europarats.

126

27.04. Aufzeichnung des Legationsrats I. Klasse von Nostitz

Nostitz erörtert einen Beitritt der Bundesrepublik zum Weltpostverein.
S. 358

S. 366

S. 343

S. 345

S. 348

S. 353

S. 355

S. 363 
Blankenhorn gibt Äußerungen des französischen Hohen Kommissars François-Poncet zur Vertretung von Berlin (West) im Bundestag und zur Stationierung französischer Truppen in Kehl wieder.

29.04. Generalkonsul Hausenstein, Paris, an das Auswärtige Amt

Hausenstein berichtet über die Positionen der Drei Mächte zu Verhandlungen mit der UdSSR.

129 30.04. Memorandum der Bundesregierung

Das Memorandum thematisiert die Einschränkung politischer Freiheitsrechte im Saargebiet.

Staatssekretärs Hallstein mit dem britischen Hohen Kommissar Kirkpatrick

Hauptthemen sind die britische Haltung zum Entwurf vom 10. März 1953 für eine Satzung der Europäischen Gemeinschaft, die Saarfrage sowie die deutschen Militärberater in Ägypten.

\section{04.05. Aufzeichnung des Vortragenden Legationsrats} von Kessel, Paris

Kessel befaßt sich mit den Ergebnissen der NATO-Ministerratstagung in Paris. von Haeften

Haeften resümiert die Gespräche, die während des Besuchs des Bundeskanzlers Adenauer in Washington über die beschlagnahmten deutschen Vermögenswerte in den USA geführt wurden.

Mosler faßt eine Unterredung mit dem schwedischen Gesandten Kumlin über die Rückgabe beschlagnahmten deutschen Vermögens zusammen. Amt

Pfeiffer berichtet über die Gespräche des Bundesministers Erhard mit dem belgischen Außenminister van Zeeland sowie Außenhandelsminister Meurice, in deren Mittelpunkt die Konvertierbarkeit der Währungen und die europäische wirtschaftliche Integration standen. 
135 06.05. Gesandter Jansen, Luxemburg, an das Auswärtige Amt

Jansen gibt Äußerungen des luxemburgischen Außenministers Bech zur Haltung der Benelux-Staaten in Fragen der europäischen Integration wieder.

136 07.05. Aufzeichnung des Ministerialdirektors Blankenhorn

Blankenhorn informiert über ein Gespräch mit dem französischen Hohen Kommissar François-Poncet. Themen waren die Unterzeichnung der Saarverträge und der Zusatzprotokolle zum EVG-Vertrag.

Bräutigam skizziert die Möglichkeiten für eine Ostpolitik der Bundesregierung.

138 11.05. Gespräch des Bundeskanzlers Adenauer mit Ministerpräsident Mayer und dem französischen Außenminister Bidault in Paris

Im Mittelpunkt stehen der Entwurf vom 10. März 1953 für eine Satzung der Europäischen Gemeinschaft und die Rede des Premierministers Churchill vom 11. Mai 1953 im britischen Unterhaus.

Kordt resümiert ein Gespräch mit dem argentinischen Botschafter Irigoyen über die Wirtschaftsverhandlungen in Buenos Aires.

140 12.05. Generalkonsul I. Klasse Krekeler, Washington, an das Auswärtige Amt

Krekeler erläutert Akzentverschiebungen in der amerikanischen Haltung zur NATO.

141 13.05. Aufzeichnung des Referenten Blomeyer-Bartenstein

Blomeyer-Bartenstein informiert über Gespräche im amerikanischen Außenministerium zur Rückgabe der Akten des Auswärtigen Amts aus der Zeit vor 1945.

142 13.05. Aufzeichnung der Legationsrätin I. Klasse von Puttkamer, z.Z. Paris

Puttkamer referiert die Stellungnahmen, die auf der Konferenz der Außenminister der EGKS-Mitgliedstaaten in Paris zum Entwurf vom 10. März 1953 für eine Satzung der Europäischen Gemeinschaft abgegeben wurden. 
143 14.05. Gespräch des Bundeskanzlers Adenauer mit Premierminister Churchill in London

Hauptthemen sind die europäische Einigung und die Rolle Großbritanniens in Europa.

144 15.05. Gespräch des Bundeskanzlers Adenauer mit

S. 429 Premierminister Churchill in London

Erörtert werden die Saarfrage, die europäische Einigung, der Besuch des Bundeskanzlers in den USA, die sowjetische Außenpolitik und die Tätigkeit deutscher Militärberater in Ägypten.

Frowein erläutert die Ansprüche auf Rückgabe bzw. Entschädigung für das in Israel befindliche deutsche Vermögen und berichtet über den Stand der Entschädigungsgesetzgebung.

Amt

Mühlenfeld berichtet über Äußerungen des Ministerpräsidenten Drees zur europäischen Verteidigung und zur geplanten Europäischen Politischen Gemeinschaft.

\section{16.05. Botschafter Kroll, Belgrad, an das Auswärtige Amt}

Kroll informiert über Ausführungen des Staatssekretärs im jugoslawischen Außenministerium, Bebler, zur Zusammenarbeit mit der NATO, zum Balkan-Pakt, zur Rede des Premierministers Churchill vom 11. Mai 1953 und zu den Wirtschaftsverhandlungen zwischen Jugoslawien und der Bundesrepublik.

Ophüls erörtert Möglichkeiten, den Verkauf der Eisenwerke Völklingen durch Maßnahmen zur wirtschaftlichen Unterstützung der Familie Röchling zu verhindern.

Salat befaßt sich mit dem Ratifizierungsverfahren von Kulturabkommen.

Kordt bittet um Klärung der Frage, inwieweit Äthiopien Interesse an einer Aufnahme diplomatischer Beziehungen hat.

Pawelke berichtet von einem Gespräch mit dem saudi-arabischen Botschafter al-Fadl über eine Aufnahme diplomatischer 
Beziehungen und über die Entsendung einer Wirtschaftsdelegation nach Djidda.

Voigt informiert über eine Demarche des iranischen Presseattachés Namdar wegen der Persiflierung von Schah Reza Pahlevi im Programm des Kabaretts „Kom(m)ödchen“. von Maltzan

Maltzan faßt die Ergebnisse der Besprechungen der Bundesminister Blücher und Erhard am 12./13. Mai 1953 in London über Währungsfragen zusammen.

Mosler informiert über die Gespräche mit dem indonesischen Gesandten Zain zum Problem des Bestehens eines Kriegszustandes zwischen Deutschland und Indonesien. Ferner seien die Beschlagnahmung deutscher Vermögenswerte und die Pensionsansprüche von ehemals im niederländischen Kolonialdienst tätigen deutschen Beamten besprochen worden.

Erörtert wird eine Stellungnahme der Bundesregierung zur Lösung der Deutschland-Frage.

Ophüls unterrichtet die Auslandsvertretungen über den Stand der Verhandlungen über die geplante Europäische Politische Gemeinschaft.

Hallstein resümiert ein Gespräch mit dem amerikanischen Ständigen Vertreter bei der EGKS, Bruce, in Paris. Im Mittelpunkt standen die Rede des Premierministers Churchill vom 11. Mai 1953, die Rede des amerikanischen Senators Taft vom 26. Mai 1953, die innenpolitische Lage in Frankreich, die europäische Einigung und das Ratifizierungsverfahren des EVGVer.trags in der Bundesrepublik.

Etzdorf informiert über den Besuch des österreichischen Außenministers Gruber in Bonn, bei dem die Errichtung einer Vertretung der Bundesrepublik in Wien sowie Eigentumsfragen thematisiert wurden. 
Strohm diskutiert die Haltung der Bundesrepublik zu Deutschland betreffenden Fragen, insbesondere zur Grenzfrage.

Vialon informiert über die Einsetzung eines Sonderausschusses des Interimsausschusses der EVG-Konferenz unter Vorsitz des belgischen Vertreters Ockrent, der sich mit dem EVG-Beitrag zum NATO-Verteidigungsprogramm befassen soll.

161 01.06. Aufzeichnung des Vortragenden Legationsrats von Etzdorf

Etzdorf resümiert ein Gespräch mit dem österreichischen Gesandten Schöner über die Aufnahme diplomatischer Beziehungen zwischen der Bundesrepublik und Österreich.

Kaufmann nimmt Stellung zum Entwurf vom 10. März 1953 für eine Satzung der Europäischen Gemeinschaft. Hallstein

Walther berichtet über die Anregung des amerikanischen Gesandten in Paris, Achilles, auch die französische Regierung über die Gespräche des Ministerialdirektors Blankenhorn in Washington zu unterrichten.

Vialon legt dar, daß im Ockrent-Ausschuß unterschiedliche Auffassungen darüber bestünden, ob nur über den finanziellen Verteidigungsbeitrag der Bundesrenuhlik für das zweite Halbjahr 1954 beraten oder aber ein einheitliches militärisches Programm der EVG erstellt werden soll.

165 03.06. Ministerialdirektor Blankenhorn, z.Z. Washington, an Bundeskanzler Adenauer und Staatssekretär Hallstein

Blankenhorn teilt mit, daß er den amerikanischen Außenminister Dulles über die Haltung der Bundesregierung zu einer Konferenz der Vier Mächte unterrichtet habe. Bundeskanzler Adenauer und Staatssekretär Hallstein

Blankenhorn berichtet über eine Unterredung mit Präsident Eisenhower anläßlich der Übergabe der beiden Schreiben des Bundeskanzlers Adenauer vom 29. und 30. Mai 1953. 
167

05.06. Aufzeichnung des Vortragenden Legationsrats von Grolmann

Grolmann erörtert völkerrechtliche Fragen im Zusammenhang mit der geplanten Entsendung eines Feldlazaretts nach Korea.

168 08.06. Aufzeichnung des Abteilungsleiters Mosler

Mosler befaßt sich mit dem französischen Wunsch, die Übergangsregelung des Anhangs $\mathrm{C}$ zum Vertrag über die Rechte und Pflichten ausländischer Streitkräfte und ihrer Mitglieder in der Bundesrepublik Deutschland zu verlängern.

169 09.06. Ministerialdirektor Blankenhorn, z.Z. Paris, an Bundeskanzler Adenauer und Staatssekretär Hallstein

Blankenhorn informiert über eine Unterredung mit dem französischen Außenminister Bidault, den er über seine Gespräche mit der amerikanischen Regierung unterrichtete.

170

10.06. Aufzeichnung des Ministerialrats Vialon, Paris

Vialon berichtet von unterschiedlichen Auffassungen der amerikanischen Regierung bzw. ihrer Vertreter bei der NATO in Paris zur Erhebung des deutschen Verteidigungsbeitrags und unterbreitet Vorschläge zum weiteren Vorgehen.

171 10.06. Vortragender Legationsrat von Etzdorf an die diplomatische Vertretung in London

Etzdorf äußert sich zur Aufnahme diplomatischer Beziehungen mit Afghanistan.

Hallstein faßt ein Gespräch mit dem französischen Stellvertretenden Hohen Kommissar Bérard zusammen. Mit Blick auf den Antrag der SPD-Fraktion vom 9. Juni 1953 nahm Bérard Stellung zu möglichen Kontakten mit dem neu ernannten sowjetischen Hohen Kommissar Semjonow und zu den innenpolitischen Veränderungen in der DDR.

Hallstein resümiert ein Gespräch mit dem französischen Stellvertretenden Hohen Kommissar Bérard über die Verlängerung der Geltungsdauer der Übergangsregelung für die französischen Streitkräfte in der Bundesrepublik, den Entwurf des Bundeswahlgesetzes und eine geplante Demonstration des „Bundes der Deutschen" in Freiburg. 
Mosler informiert über eine Unterredung mit dem Unterstaatssekretär im spanischen Außenministerium, Navascués, zur Freigabe des deutschen Vermögens in Spanien.

Pauls gibt Äußerungen des französischen Stellvertretenden Hohen Kommissars Bérard zu einer Kontaktaufnahme mit dem sowjetischen Hohen Kommissar Semjonow und zu den innenpolitischen Veränderungen in der DDR wieder.

Oberbefehlshaber der NATO-Streitkräfte in Europa,

\section{Ridgway}

Im Mittelpunkt stehen die EVG, die bevorstehenden Bundestagswahlen, die Bemühungen um die europäische Einigung und die Einschätzung der sowjetischen Außenpolitik.

\section{z.Z. Dublin}

Scherpenberg resümiert die Verhandlungen vom 12. bis 15 . Juni 1953 in Dublin über die Entschädigung für Verluste und Schäden aus dem Zweiten Weltkrieg.

Meyer berichtet über Gespräche mit dem Staatssekretär im indischen Außenministerium, Nehru, und dem türkischen Botschafter Seynen zu den außenpolitischen Initiativen der UdSSR. das Auswärtige Amt

Meynen gibt eine Einschätzung der sowjetischen Politik gegenüber der DDR.

Hallstein faßt ein Gespräch mit dem schwedischen Gesandten Kumlin über Ausführungen des schwedischen Außenministers Undén zur Deutschland-Frage zusammen.

Krapf gibt eine Stellungnahme des Referatsleiters im französischen Außenministerium, Sauvagnargues, zur sowjetischen Deutschlandpolitik wieder. 
Allardt informiert über Besprechungen mit Botschafter Pawelke, Kairo, und den deutschen Militärexperten in Ägypten. von Etzdorf

Etzdorf legt eine Einschätzung der sowjetischen Außenpolitik vor.

184 17.06. Aufzeichnung des Gesandten I. Klasse Ophüls

Ophüls berichtet von einem Gespräch mit dem amerikanischen Beobachter beim Interimsausschuß der EVG-Konferenz, Bruce, über die Ratifizierung des EVG-Vertrags, die geplante Europäische Politische Gemeinschaft und eine mögliche Vier-Mächte-Konferenz.

17.06. Aufzeichnung des Vortragenden Legationsrats von Etzdorf

Etzdorf resümiert eine Unterredung mit dem Direktor der Ruhrverbände, Prüß, über das Assuan-Staudamm-Projekt.

186 17.06. Aufzeichnung des Staatssekretärs Hallstein

Hallstein faßt Mitteilungen des französischen Stellvertretenden Hohen Kommissars Bérard über die Situation in Berlin zusammen. das Auswärtige Amt

Meynen berichtet über die Demonstrationen am 16./17. Juni 1953 in Ost-Berlin.

188 18.06. Aufzeichnung des Ministerialrats Vialon, Paris

Vialon gibt eine Diskussion im Ockrent-Ausschuß über den EVG-Beitrag zur NATO wieder.

189 18.06. Aufzeichnung des Vortragenden Legationsrats Thierfelder

Thierfelder referiert den Stand der Überlegungen hinsichtlich einer finanziellen Stützungsaktion zugunsten der Familie Röchling, um den Verkauf der Eisenwerke in Völklingen zu verhindern.

\section{20.06. Aufzeichnung des Staatssekretärs Hallstein}

Hallstein informiert über eine Unterredung mit dem französischen Hohen Kommissar François-Poncet, in deren Mittelpunkt 
die Demonstrationen am 16./17. Juni 1953 in Ost-Berlin sowie die Erhöhung der Stärke des Bundesgrenzschutzes standen.

Hallstein übermittelt eine Sprachregelung zu den Ereignissen vom 17. Juni 1953 in Ost-Berlin und der DDR. Staatssekretär Hallstein

Meynen erteilt Auskunft über die Beziehungen zwischen den Stadtkommandanten der Drei Mächte und den sowjetischen Behörden in Berlin.

Hake berichtet von einem Gespräch mit dem Kabinettschef des französischen Residenten in Rabat, Baudouy, über die Errichtung einer Vertretung der Bundesrepublik in Marokko. Auswärtige Amt

Krekeler informiert über ein amerikanisches Aide-mémoire bezüglich der Haltung der Bundesrepublik zum Embargo gegenüber der Volksrepublik China.

Vialon referiert die Diskussion im Lenkungsausschuß der EVGKonferenz über die Verhandlungen im Ockrent-Ausschuß. Trützschler von Falkenstein

Trützschler faßt ein Gespräch mit Ministerpräsident Nehru über die deutschen Kriegsgefangenen in der UdSSR zusammen.

Vialon gibt die Anregung des amerikanischen stellvertretenden Beobachters beim Interimsausschuß der EVG-Konferenz, Tomlinson, weiter, bei Gesprächen mit der amerikanischen Regierung über die Verteidigungsleistungen der EVG unbedingt am Vorschlag des Ockrent-Ausschusses festzuhalten.

Adenauer nimmt zu den französisch-saarländischen Verträgen vom 20. Mai 1953 Stellung. 
Biermann äußert sich zu den Verhandlungen über die Bereitstellung eines Feldlazaratts für amerikanische Truppen in Korea.

Born resümiert Gespräche über die Einsetzung eines Gemischten Ausschusses zur Begnadigung wegen Kriegsverbrechen verurteilter Deutscher, die sich im Gewahrsam der Drei Mächte befinden.

Biermann befaßt sich mit der Frage eines zusätzlichen Beitrags der Bundesrepublik zum Hilfsprogramm der UNO für arabische Palästina-Flüchtlinge.

Ophüls erörtert, ob Frankreich nach Inkrafttreten des EVG-Vertrags noch Truppen im Saargebiet unterhalten dürfe.

Simon thematisiert die Möglichkeit einer Rückgabe der Büste der Nofretete an Ägypten.

Mosler legt die Schwierigkeiten dar, die sich durch die Zusammensetzung von Auslandsdelegationen aus Vertretern verschiedener Ressorts für die Verhandlungsführung ergeben.

Krekeler berichtet von amerikanischen Überlegungen, wie auf die Ereignisse vom 17. Juni 1953 in Ost-Berlin und in der DDR reagiert werden könnte. Er befaßt sich außerdem mit der bevorstehenden Außenministerkonferenz der Drei Mächte in Washington.

Blankenhorn faßt ein Gespräch des Bundeskanzlers Adenauer mit dem amerikanischen Hohen Kommissar Conant über die bevorstehende Außenministerkonferenz der Drei Mächte in Washington zusammen.

Pawelke gibt die Information weiter, daß von britischer Seite Bedenken gegen die Errichtung einer Gesandtschaft der Bundesrepublik in Libyen bestünden. 

Trützschler von Falkenstein

Trützschler befürwortet die Zahlung eines Beitrags für das Jahr 1953 an das Weltkinderhilfswerk UNICEF. Trützschler von Falkenstein

Trützschler stellt den Stand der Verhandlungen über die Rückführung der von den Drei Mächten beschlagnahmten Akten des Auswärtigen Amts aus der Zeit vor 1945 dar.

Hallstein resümiert eine Unterredung mit dem Politischen Berater im amerikanischen Hochkommissariat, Steere, über die geplante Lebensmittelaktion für die DDR, die bevorstehende Außenministerkonferenz der Drei Mächte in Washington und die Anwerbung nicht-deutscher Freiwilliger im Bundesgebiet für die amerikanische Armee.

211 07.07. Aufzeichnung des Ministerialdirektors Blankenhorn

Blankenhorn gibt ein Gespräch mit dem Staatssekretär wieder, in dem er mit Hallstein den Vorschlag einer Vier-Mächte-Konferenz über die Deutschland-Frage sowie Möglichkeiten für Angebote an die UdSSR zur Förderung der Sicherheit in Europa erörterte.

212 07.07. Botschafter Kroll, Belgrad, an das Auswärtige Amt

Kroll berichtet von einem Gespräch mit dem jugoslawischen Vizepräsidenten Kardelj über den Stand der Wirtschaftsverhandlungen. das Auswärtige Amt

Meynen informiert über den Besuch des amerikanischen Hohen Kommissars Conant in Berlin (West) und über Äußerungen des Vertreters der amerikanischen Hohen Kommission in Berlin, Lyon, zur sowjetischen Deutschland-Politik.

Hausenstein gibt Äußerungen des französischen Außenministers Bidault zur kommenden Außenministerkonferenz der EGKSMitgliedstaaten in Baden-Baden wieder. 
Adenauer schlägt der bevorstehenden Außenministerkonferenz in Washington ein Sofortprogramm zur Wiederherstellung der Freiheiten in ganz Deutschland vor.

216 10.07. Aufzeichnung des Vortragenden Legationsrats Trützschler von Falkenstein

Trützschler befaßt sich mit dem amerikanischen Wunsch, im Bundesgebiet nicht-deutsche Freiwillige für die amerikanische Armee anzuwerben. Auswärtige Amt

Knoke berichtet von einem Gespräch mit dem griechischen Koordinationsminister Markesinis über Themen für dessen Besuch in der Bundesrepublik.

Blankenhorn informiert über eine Unterredung mit dem Abteilungsleiter im amerikanischen Außenministerium, Riddleberger, anläßlich der Übergabe des Schreibens des Bundeskanzlers Adenauer an den amerikanischen Außenminister Dulles mit dem Vorschlag einer Vier-Mächte-Konferenz. Staatssekretär Hallstein

Blankenhorn übermittelt den amerikanischen Entwurf für eine Deutschland-Erklärung der Außenministerkonferenz der Drei Mächte in Washington.

Hassell legt dar, inwieweit die EVG dem Sicherheitsbedürfnis auch von Nicht-Mitgliedstaaten Rechnung trägt, und unterbreitet Vorschläge für eine zusätzliche Nichtangriffsgarantie der EVG an die UdSSR.

Blankenhorn informiert über eine Stellungnahme des Unterstaatssekretärs im britischen Außenministerium, Roberts, zu 
der von Bundeskanzler Adenauer vorgeschlagenen Vier-MächteKonferenz.

Herwarth resümiert ein Gespräch des Staatssekretärs Hallstein mit dem österreichischen Botschafter Schmid. Themen waren die Einfuhr von Käse aus Österreich und die Errichtung einer Vertretung der Bundesrepublik in Wien.

Hallstein regt eine Ressortbesprechung über den möglichen Verkauf der Eisenwerke in Völklingen durch die Familie Röchling an und übermittelt eine Aufzeichnung über den Sachstand.

Hausenstein faßt ein Gespräch mit dem ehemaligen französischen Außenminister Schuman über die EVG und die Einschätzung der UdSSR zusammen.

Kielmansegg nimmt zu den Überlegungen des Legationsrats von Hassell über die EVG als Sicherheitssystem Stellung.

Kordt weist die Botschaft an, dem äthiopischen Geschäftsträger die Bereitschaft der Bundesregierung zur Wiederaufnahme diplomatischer Beziehungen mitzuteilen.

Etzdorf legt die Schwierigkeiten bei der Errichtung einer Handelsvertretung in Wien dar. von Kessel

Nach einem Aufenthalt in den USA schildert Kessel Eindrücke über die Einsatzfähigkeit der amerikanischen Streitkräfte, die amerikanische Innenpolitik, die Haltung im Korea-Krieg und die Beziehungen der USA zu Großbritannien und Frankreich sowie zur UdSSR und zur Bundesrepublik.

229 24.07. Botschafter Pawelke, Kairo, an das Auswärtige Amt

Pawelke informiert über Gespräche mit dem amerikanischen Botschafter Caffery und dem ägyptischen Vizepräsidenten Nasser, in denen Lösungsmöglichkeiten für den Konflikt zwischen Großbritannien und Ägypten um die britischen Stützpunkte am Suez-Kanal erörtert wurden. 
Klee berichtet über den Wunsch der guatemaltekischen Regierung, ein Abkommen mit der Bundesrepublik über die Beendigung des Kriegszustands und die Normalisierung der Beziehungen zu schließen. Kommissar François-Poncet

Hallstein übermittelt Vorstellungen des Bundeskanzlers Adenauer zu den Zielen und Aufgaben der bevorstehenden Außenministerkonferenz der EGKS-Mitgliedstaaten in Baden-Baden über eine europäische politische Integration.

Mit Blick auf eine Vier-Mächte-Konferenz erörtert Bräutigam die sowjetische Interessenlage und die sich daraus ergebenden Schlußfolgerungen für die Politik der westeuropäischen Staaten.

Walther informiert über die französische Reaktion auf Überlegungen des Bundeskanzlers Adenauer, die EVG zum Ausgangspunkt eines Sicherheitssystems in Europa zu machen. das Auswärtige Amt

Meynen berichtet von einem Gespräch mit dem Vorsitzenden des Rates der Evangelischen Kirchen in Deutschland, Dibelius, über die Situation in Ost-Berlin und der DDR. Washington

Sigrist faßt die Gespräche des Sonderbeauftragten des Bundeskanzlers, Blank, im amerikanischen Außenministerium zusammen, in deren Mittelpunkt die Planungen für den Aufbau der EVG-Kontingente standen. Auswärtige Amt

Schlange-Schöningen informiert über die Absicht des amtierenden britischen Außenministers Lord Salisbury, einen Sicherheitsplan unter Berücksichtigung sowjetischer Interessen auszuarbeiten.

Blankenhorn unterbreitet einen Vorschlag für ein europäisches Sicherheitssystem unter Einbeziehung der UdSSR. 
Frowein informiert über ein Gespräch mit dem stellvertretenden Leiter der Israel-Mission, Jachil. Erörtert wurden Wiedergutmachungsfragen sowie die Wahrnehmung von konsularischen Befugnissen durch die Israel-Mission.

Hallstein berichtet über die Vorbereitungen zur Außenministerkonferenz der EGKS-Mitgliedstaaten in Baden-Baden.

240 07.08. Vortragender Legationsrat von Kessel, Paris, an das Auswärtige Amt

Kessel resümiert Gespräche mit dem Stellvertretenden NATOGeneralsekretär van Vredenburch. Themen waren die Bundestagswahl, die EVG und die Wiedervereinigung Deutschlands.

241 10.08. Gespräch des Bundeskanzlers Adenauer mit dem französischen Außenminister Bidault

Erörtert werden die Saarfrage, der Verteidigungsbeitrag der Bundesrepublik und die Fortsetzung der europäischen Integration. Vertretung in London

Hallstein erläutert Bedenken gegen Überlegungen der Drei Mächte zu einer Antwort auf die sowjetische Note vom 4. August 1953.

\section{13.08. Aufzeichnung des Vizekonsuls a. D. de Haas, Kairo}

De Haas berichtet von einer Unterredung mit dem saudi-arabischen Außenminister Prinz Feisal über die Aufnahme diplomatischer Beziehungen. Auswärtige Amt

Schlange-Schöningen resümiert Unterredungen mit Vertretern der Drei Mächte über eine Vier-Mächte-Konferenz.

Scherpenberg informiert über die Beteiligung eines Konsortiums aus der Bundesrepublik am Aufbau eines Stahlwerkes in Indien. 
Hallstein berichtet über ein Gespräch mit dem französischen Stellvertretenden Hohen Kommissar Bérard, in dessen Mittelpunkt die französische Reaktion auf die sowjetische Note vom 15. August 1953 stand.

Hallstein übermittelt eine Sprachregelung zur sowjetischen Note vom 15. August 1953.

Hallstein weist Pawelke an, im Konflikt zwischen Großbritannien und Ägypten um britische Stützpunkte am Suez-Kanal eine neutrale Haltung zu bewahren.

Blankenhorn informiert die Auslandsvertretungen über die Außenministerkonferenz der EGKS-Mitgliedstaaten in Baden-Baden. Hallstein

Hausenstein übermittelt Außerungen des Referatsleiters im amerikanischen Außenministerium, Morris, zu Verhandlungen der Drei Mächte über die Antwort auf die sowjetischen Noten vom 4. und 15. August 1953. Amt

Krekeler berichtet über ein Gespräch mit dem Abteilungsleiter im amerikanischen Außenministerium, Merchant. Erörtert wurden die Antwort der Drei Mächte auf die sowjetischen Noten vom 4. und 15. August 1953, die Ratifizierung des EVG-Vertrags und die Saarfrage. Generalsekretär des Europarats, Lincoln

Hallstein nimmt Stellung zur Einbeziehung des Saargebiets in die Konventionen des Europarats. Hallstein

Hausenstein übermittelt Äußerungen des Abteilungsleiters im französischen Außenministerium, Laloy, zu den Verhandlungen der Drei Mächte über eine Antwort auf die sowjetischen Noten vom 4. und 15. August 1953. 
Hallstein faßt Gespräche mit Vertretern der Drei Mächte über die Antwort auf die sowjetischen Noten vom 4. und 15. August 1953 zusammen.

Amt

Koenning erläutert den Stand der Beziehungen zu Finnland.

Kaumann bittet um Weisung hinsichtlich der Aufnahme von diplomatischen Beziehungen zu den Philippinen.

Blankenhorn berichtet von einer Unterredung des Bundeskanzlers Adenauer mit dem amerikanischen Hohen Kommissar Conant über die Antwort der Drei Mächte auf die sowjetischen Noten vom 4. und 15. August 1953.

Hallstein faßt ein Gespräch des Bundeskanzlers Adenauer mit dem französischen Hohen Kommissar François-Poncet zusammen. Themen waren u. a. die Saarfrage, die Antwort der Drei Mächte auf die sowjetischen Noten vom 4. und 15. August 1953, die Verstärkung des Bundesgrenzschutzes, die Bundestagswahl und die Aufhebung des Interzonenpaßzwangs.

02.09. Botschafter Schlange-Schöningen, London, an das Auswärtige Amt

Schlange-Schöningen informiert über Gespräche mit Vertretern der Drei Mächte. Erörtert wurde die Antwort auf die sowjetischen Noten vom 4, und 15. August 1953. Auswärtige Amt

Kessel berichtet von Überlegungen innerhalb der NATO zu einem Verteidigungsbeitrag der Bundesrepublik und zur Wiedervereinigung Deutschlands.

Bidder äußert sich zur Frage einer Einschaltung der USA in die Bemühungen um Aufnahme von diplomatischen Beziehungen zu Äthiopien. 
262

09.09. Aufzeichnung des Ministerialdirektors Blankenhorn

Blankenhorn resümiert ein Gespräch des Bundeskanzlers Adenauer mit dem amerikanischen Beobachter beim Interimsausschuß der EVG-Konferenz, Bruce. Erörtert wurden die Fortführung der Amtsgeschäfte des Außenministers durch Adenauer, die Saarfrage und die Ratifizierung des EVG-Vertrags.

09.09. Aufzeichnung des Botschaftsrats a.D. Hilger

S. 792

Hilger gibt eine Einschätzung der sowjetischen Deutschlandpolitik.

264 10.09. Generalkonsul Riesser, New York (UNO), an das Auswärtige Amt

Riesser informiert über ein Gespräch mit UNO-Generalsekretär Hammarskjöld, der von einem Meinungsaustausch mit dem sowjetischen Stellvertretenden Außenminister Wyschinskij über die Rückführung von deutschen Kriegsgefangenen aus der Sowjetunion berichtete.

11.09. Gespräch des Bundeskanzlers Adenauer mit dem amerikanischen Hohen Kommissar Conant

Erörtert werden die Themen einer Vier-Mächte-Konferenz über Deutschland.

12.09. Aufzeichnung des Ministerialdirigenten von Etzdorf

S. 797

Etzdorf äußert sich zur Errichtung einer Vertretung der Bundesrepublik in Österreich.

Hallstein faßt ein Gespräch mit dem Präsidenten der Hohen Behörde der EGKS, Monnet, zusammen. Themen waren die französische Haltung zur EVG und zu einer Europäischen Politischen Gemeinschaft, die militärische Präsenz der USA in Europa, die britische Einstellung gegenüber der europäischen Integration sowie die Haltung der UdSSR zu einer deutschen Armee.

14.09. Botschafter Hausenstein, Paris, an das Auswärtige Amt

Hausenstein informiert über ein Gespräch mit dem Geschäftsträger der Republik China (Taiwan) in Paris, Tuan Mao-lan, in dem die Intensivierung der bilateralen Beziehungen zwischen der Republik China (Taiwan) und der Bundesrepublik erörtert wurde. 

Auswärtige Amt

Kessel berichtet von einem Gespräch mit dem Stellvertretenden NATO-Generalsekretär Vredenburch über die Probleme der Ratifizierung des EVG-Vertrags durch Frankreich.

Blankenhorn resümiert ein Gespräch mit dem französischen Außenminister Bidault über die Ratifizierung des EVG-Vertrags, eine Europäische Politische Gemeinschaft und eine VierMächte-Konferenz über Deutschland. Thierfelder

Thierfelder informiert über ein Gespräch mit dem Berichterstatter des Ausschusses für Allgemeine Angelegenheiten der Beratenden Versammlung des Europarats. Erörtert wurden wirtschaftliche Aspekte im Saarbericht von van der Goes van Naters.

Hallstein erläutert eine Erklärung des Bundeskanzlers Adenauer über ein europäisches Sicherheitssystem und erteilt Weisung für ihre Rücknahme.

Blankenhorn berichtet über Gespräche mit französischen Regierungsvertretern, in denen eine Europäische Politische Gemeinschaft, ein möglicher Gedankenaustausch des Bundeskanzlers Adenauer mit dem französischen Außenminister Bidault, die Ratifizierung des EVG-Vertrags und eine Vier-Mächte-Konferenz über Deutschland thematisiert wurden. Auswärtige Amt

Schlange-Schöningen übermittelt Informationen aus einem Gespräch mit einem Mitarbeiter der amerikanischen Botschaft in London zu Vorschlägen für ein Sicherheitssystem unter Einbeziehung der UdSSR.

Hallstein unterrichtet über den Beginn der Konferenz der Stellvertreter der Außenminister der EGKS-Mitgliedstaaten in Rom über eine Europäische Politische Gemeinschaft. 

van Scherpenberg

Scherpenberg berichtet über die Bemühungen, im Rahmen von Verhandlungen über ein Warenabkommen die Rückführung von Deutschen aus Polen zu thematisieren.

Feine gibt Informationen des türkischen Prinzen Ekrem wieder, wonach Israel und die UdSSR über den Ankauf deutscher Dollaranleihen verhandelten, die von der UdSSR nach Beendigung des Zweiten Weltkriegs beschlagnahmt wurden.

Etzdorf resümiert ein Gespräch mit dem französischen Stellvertretenden Hohen Kommissar Bérard. Eine gemischte Kommission solle Bedingungen für eine Wiedervereinigung Deutschlands prüfen.

Melchers berichtet über ein Gespräch mit Ministerpräsident Fadhil el-Jamali, in dessen Mittelpunkt die arabische Politik gegenüber Israel und die Lage der Flüchtlinge aus Palästina standen. Thierfelder

Thierfelder erörtert das Antwortschreiben des französischen AuBenministers Bidault auf die Note der Bundesregierung zu den Verträgen vom 20. Mai 1953 zwischen Frankreich und dem Saarland. Amt

Walther berichtet von einem Gespräch mit dem Referatsleiter im französischen Außenministerium, de Margerie, über die sowjetische Note vom 28 . September 1953, über den Stand der Ratifizierung des EVG-Vertrags in Frankreich und die Saarfrage.

Grewe nimmt Stellung zum Antwortschreiben des französischen Außenministers Bidault auf die Note der Bundesregierung zu den Verträgen vom 20. Mai 1953 zwischen Frankreich und dem Saarland. 

Thierfelder

Thierfelder äußert sich zur Hissung der saarländischen Flagge anläßlich eines Fußballqualifikationsspiels zwischen einer Auswahl der Bundesrepublik und des Saarlandes in Stuttgart.

Hendus faßt Ergebnisse der Konferenz der Stellvertreter der Außenminister der EGKS-Mitgliedstaaten in Rom über eine Europäische Politische Gemeinschaft zusammen. Thierfelder

Thierfelder erörtert den Saarbericht des Berichterstatters des Ausschusses für Allgemeine Angelegenheiten der Beratenden Versammlung des Europarats, van der Goes van Naters.

03.10. Vortragender Legationsrat von Kessel, Paris, an das Auswärtige Amt

Kessel teilt mit, wie in Kreisen des Interimsausschusses der EVG-Konferenz die Möglichkeiten für eine rasche Ratifizierung des EVG-Vertrags eingeschätzt werden.

05.10. Gespräch des Bundeskanzlers Adenauer mit den Hohen Kommissaren Conant und Hoyer Millar sowie dem Stellvertretenden Hohen Kommissar Bérard

Themen sind die Antwort der Drei Mächte auf die sowjetische Note vom 28. September 1953, die Ratifizierung des EVG-Vertrags und die Rückführung deutscher Kriegsgefangener aus der UdSSR.

Etzdorf resümiert eine Direktorenbesprechung, in der das künftige Verfahren bei der Restitution italienischer Kunstwerke erörtert wurde.

Bräutigam notiert Ergebnisse der zweiten Sitzung der Arbeitsgruppe "Wiedervereinigung“ über die Durchführung gesamtdeutscher Wahlen.

Trützschler informiert über ein Gespräch mit dem irakischen Gesandten Khandan. Thema war ein Boykott von Firmen aus der Bundesrepublik, die Waren an Israel liefern. 
291 12.10. Aufzeichnung des Referenten Oncken

S. 866

Oncken faßt eine Besprechung mit Vertretern der Drei Mächte zum Aide-mémoire der AHK vom 22. September 1953 über die Durchführung gesamtdeutscher Wahlen zusammen.

292 12.10. Ministerialdirektor Blankenhorn an Botschafter

S. 872 Schlange-Schöningen, London

Blankenhorn erteilt Weisung zu einem Themenvorschlag für eine Vier-Mächte-Konferenz, der die Deutschlandfrage betrifft.

Frowein berichtet von einem Gespräch mit dem stellvertretenden Leiter der Israel-Mission, Jachil, über die Wahrnehmung von konsularischen Befugnissen durch die Israel-Mission.

294 13.10. Botschafter Schlange-Schöningen, London, an Ministerialdirektor Blankenhorn

Schlange-Schöningen informiert über eine Unterredung mit dem Abteilungsleiter im britischen Außenministerium, Allen, zur Antwort der Drei Mächte auf die sowjetische Note vom 28. September 1953.

295 14.10. Gespräch des Bundeskanzlers Adenauer mit dem amerikanischen Beobachter beim Interimsausschuß der EVG-Konferenz, Bruce

Erörtert werden Planungen für eine Europäische Politische Gemeinschaft und die Saarfrage. Auswärtige Amt

Kessel berichtet von einem Gespräch mit dem Vorsitzenden des Interimsausschusses der EVG-Konferenz, Alphand, über die Ratifizierung des EVG-Vertrags und ein europäisches Sicherheitssystem. belgischen Außenminister van Zeeland in Rhöndorf

Themen sind die Überlegungen von van Zeeland für ein europäisches Sicherheitssystem und für eine Wiedervereinigung Deutschlands.

Meissner erörtert die Möglichkeiten für ein europäisches Sicherheitssystem unter Einbeziehung der UdSSR. 
Hallstein unterrichtet über ein Gespräch mit dem Unterstaatssekretär im britischen Außenministerium, Roberts. Im Mittelpunkt standen die Ratifizierung des EVG-Vertrags, die Saarfrage, ein europäisches Sicherheitssystem und die Vorbereitungen der Drei Mächte für eine Konferenz mit der UdSSR.

300

301

302

303

20.10. Botschafter Schlange-Schöningen, London, an das Auswärtige Amt

Schlange-Schöningen informiert über die Außenministerkonferenz der Drei Mächte in London, die Antwort auf die sowjetische Note vom 28. September 1953 und die Triest-Frage.

21.10. Staatssekretär Hallstein, z.Z. Washington, an

Bundeskanzler Adenauer und Ministerialdirektor Blankenhorn

Hallstein berichtet über ein Gespräch mit dem amerikanischen Außenminister Dulles. Themen waren die Ratifizierung des EVG-Vertrags, die Überlegungen des belgischen Außenministers van Zeeland für ein europäisches Sicherheitssystem, die Beendigung des Besatzungsstatuts und die Konsultation der Bundesregierung vor einer Vier-Mächte-Konferenz.

\subsection{Aufzeichnung des Gesandtschaftsrats von Török}

S. 898

Török resümiert eine Ressortbesprechung über die Befugnisse, die eine aus gesamtdeutschen Wahlen hervorgegangene Nationalversammlung haben sollte.

\subsection{Aufzeichnung des Referenten Oncken}

S. 901

Oncken erläutert die unterschiedlichen Auffassungen der Ressorts zu den Befugnissen, die einer aus gesamtdeutschen Wahlen hervorgegangenen Nationalversammlung zustehen sollten.

24.10. Aufzeichnung des Rechtsberaters Kaufmann

Kaufmann unterbreitet einen Vorschlag zur Beantwortung der Fragen 1 bis 15 des Aide-mémoire der AHK vom 22. September 1953 über die Durchführung gesamtdeutscher Wahlen.

24.10. Generalkonsul Feine, Genf, an das Auswärtige Amt

Feine berichtet über Vorstöße für eine Beteiligung der DDR an den Arbeiten der ECE. 
Fischer erörtert die Beziehungen zur Republik China (Taiwan) und die Möglichkeit von Wirtschaftsverhandlungen mit der Volksrepublik China. Auswärtige Amt

Kessel informiert über ein Gespräch mit dem Stellvertretenden NATO-Generalsekretär van Vredenburch. Im Mittelpunkt standen eine mögliche Verminderung der amerikanischen Truppen in Europa, die Ratifizierung des EVG-Vertrags und ein Verteidigungsbeitrag der Bundesrepublik im Rahmen der NATO.

Hallstein legt einen Vorschlag für ein Saarstatut vor.

Hallstein begründet und erläutert seinen Vorschlag für ein Saarstatut.

310 31.10. Aufzeichnung des Gesandtschaftsrats I. Klasse Böker, Paris

Böker berichtet von einem Gespräch mit dem stellvertretenden Abteilungsleiter im britischen Außenministerium, Warner, über die Vorbereitungen der Drei Mächte für eine Konferenz mit der UdSSR.

311 31.10. Aufzeichnung des Rechtsberaters Kaufmann

Kaufmann unterbreitet einen Vorschlag zur Beantwortung der Fragen 16 bis 20 des Aide-mémoire der AHK vom 22. September 1953 über die Bildung einer gesamtdeutschen Regierung.

312 03.11. Gespräch des Bundeskanzlers Adenauer mit dem französischen Hohen Kommissar François-Poncet

Die Gesprächspartner erörtern Möglichkeiten einer Lösung der Saarfrage.

313 03.11. Aufzeichnung des Generalleutnants a.D. Speidel, Paris

Speidel resümiert ein Gespräch mit dem italienischen Delegationsleiter beim Interimsausschuß der EVG-Konferenz, Lombardo, über die Ratifizierung des EVG-Vertrags und die TriestFrage. 

Hallstein

Hausenstein berichtet über die Besprechungen der Drei Mächte zur Vorbereitung einer Konferenz mit der UdSSR. Jansen übermittelt Äußerungen des luxemburgischen Außenministers Bech über die französische Haltung zur EVG.

Bräutigam analysiert die sowjetische Note vom 3 . November 1953 an die Drei Mächte.

\section{06.11. Aufzeichnung des Staatssekretärs Hallstein}

Hallstein faßt eine Unterredung mit dem amerikanischen Stellvertretenden Hohen Kommissar Dowling zusammen. Themen waren die Anwerbung von nicht-deutschen Freiwilligen für die amerikanische Armee im Bundesgebiet, die sowjetische Note vom 3. November 1953 und die Aufhebung des Interzonenpaßzwanges.

318 10.11. Vortragender Legationsrat von Kessel, Paris, an Ministerialdirigent van Scherpenberg

Kessel informiert über die Beteiligungsmöglichkeiten für Firmen aus der Bundesrepublik an Ausschreibungen für den Bau von Flugplätzen in der Türkei.

319 12.11. Runderlaß des Gesandten I. Klasse Strohm

Strohm erteilt Weisung, Kontakte zu den Vertretungen der osteuropäischen Staaten und der Volksrepublik China zu unterlassen. Trützschler von Falkenstein

Trützschler legt den Stand der Beratungen zur Bereitstellung eines Feldlazaretts für Korea dar.

321 13.11. Botschafter Haas, Ankara, an das Auswärtige Amt

Haas berichtet über ein Gespräch mit Ministerpräsident Menderes. Themen waren die Eingliederung der Bundesrepublik in die atlantische Verteidigungsgemeinschaft, Verhandlungen der Drei Mächte mit der UdSSR und die Rückgabe deutschen Eigentums. 
Speidel resümiert ein Gespräch mit dem amerikanischen Beobachter beim Interimsausschuß der EVG-Konferenz, Bruce, zur Saarfrage und zur Ratifizierung des EVG-Vertrags in Frankreich.

Blankenhorn unterbreitet eine Einschätzung der außenpolitischen Lage und der Entwicklung der UdSSR. Hallstein

Hausenstein übermittelt Äußerungen des amerikanischen Beobachters beim Interimsausschuß der EVG-Konferenz, Bruce, zur Saarfrage. Thierfelder, z.Z. Den Haag

Thierfelder berichtet über ein Gespräch mit dem Berichterstatter des Ausschusses für Allgemeine Angelegenheiten der Beratenden Versammlung des Europarats, van der Goes van Naters, zu dessen Saarbericht.

Oberbefehlshaber der NATO-Streitkräfte in Europa, Gruenther

Themen sind die Ratifizierung des EVG-Vertrags in Frankreich, die Bedrohung Europas und der USA durch die UdSSR, die bevorstehende Außenministerkonferenz der EGKS-Mitgliedstaaten über eine Europäische Politische Gemeinschaft sowie mögliche Reaktionen der USA bei einem Scheitern der EVG.

Ophüls faßt ein Gespräch des Staatssekretärs Hallstein mit dem niederländischen Außenminister Beyen zu Fragen einer Europäischen Politischen Gemeinschaft zusammen.

amerikanischen Beobachter beim Interimsausschuß der EVG-Konferenz, Bruce

Im Mittelpunkt stehen die vorbereitenden Gespräche des Bundeskanzlers mit dem französischen Hohen Kommissar François-Poncet zur Lösung der Saarfrage. 

Adenauer und Staatssekretär Hallstein

Krekeler berichtet über ein Gespräch mit dem Staatssekretär im amerikanischen Außenministerium, Smith. Themen waren die amerikanische Truppenstärke in Europa, die Ratifizierung des EVG-Vertrags, die Saarfrage und die Konsultation der Bundesregierung während der Konferenz der Staats- und Regierungschefs der Drei Mächte auf den Bermudas.

331 19.11. Aufzeichnung des Staatssekretärs Hallstein

Hallstein resümiert eine Unterredung des Bundeskanzlers Adenauer mit dem französischen Hohen Kommissar François-Poncet und dem französischen Stellvertretenden Hohen Kommissar Bérard über die Unterzeichnung der Zusatzprotokolle zum EVGVertrag.

332 19.11. Botschafter Pawelke, Kairo, an das Auswärtige Amt

Pawelke informiert über die Errichtung eines Handelsbüros der DDR in Ägypten.

Hallstein macht Vorschläge zur Abgrenzung der Zuständigkeiten zwischen Auswärtigem Amt und Bundesministerium des Innern auf kulturpolitischem Gebiet.

334 21.11. Aufzeichnung des Gesandten I. Klasse Ophüls

Ophüls übermittelt Äußerungen des amerikanischen Botschaftssekretärs in Paris, Cleveland, zur innenpolitischen Lage in Frankreich und zur Position der USA in der Saarfrage.

21.11. Botschafter Clemens von Brentano, Rom, an das Auswärtige Amt

Brentano berichtet über ein Gespräch mit Ministerpräsident Pella zur Triest-Frage und zu Presseäußerungen in der Bundesrepublik über Südtirol. Blank

Blank nimmt Stellung zur Unterzeichnung von Zusatzprotokollen zum EVG-Vertrag.

Vorschläge zur Lösung der Saarfrage werden unterbreitet. 
338

26.11. Gesandter Mueller-Graaf, z.Z. Wien, an das Auswärtige Amt

Mueller-Graaf berichtet über einen Besuch bei Bundeskanzler Raab und dem österreichischen Außenminister Figl.

26.11. Botschaftsrat Freiherr von Mirbach, Kairo, an das Auswärtige Amt

Mirbach informiert über ein Gespräch mit dem Abteilungsleiter im ägyptischen Außenministerium, Aboul Fetouh, zum Status eines Handelsbüros der DDR in Kairo.

27.11. Bundeskanzler Adenauer an Botschafter Krekeler, Washington

Adenauer läßt Bundesminister Erhard ersuchen, sich während seines Aufenthaltes in den USA zurückhaltend zur Frage der Währungskonvertibilität zu äußern.

27.11. Gesandter Mueller-Graaf, z.Z. Wien, an das Auswärtige Amt

Mueller-Graaf berichtet über Besuche beim Staatssekretär im österreichischen Außenministerium, Kreisky, und bei Vizekanzler Schärf, in deren Mittelpunkt die Errichtung einer Handelsvertretung der Bundesrepublik stand.

28.11. Gespräch des Bundeskanzlers Adenauer mit dem französischen Außenminister Bidault in Den Haag

Die Gesprächspartner erörtern französische Vorschläge zur Saarfrage.

Welck übermittelt eine Sprachregelung des Staatssekretärs Hallstein zur sowjetischen Note vom 26. November 1953.

344 30.11. Aufzeichnung des Referenten Oncken

Oncken analysiert das Schreiben des amerikanischen Hohen Kommissars Conant an Bundeskanzler Adenauer zur Durchführung gesamtdeutscher Wahlen und zur Bildung einer gesamtdeutschen Regierung.

345 30.11. Bundeskanzler Adenauer an Staatssekretär Hallstein, z.Z. Washington

Adenauer unterrichtet über eine Unterredung mit dem amerikanischen Hohen Kommissar Conant zur sowjetischen Note vom 26. November 1953.
S. 1011

S. 1012

S. 1014

S. 1017

S. 1025 
346 Nov. Aufzeichnung des Bundeskanzlers Adenauer

Die sowjetische Außenpolitik wird analysiert.

347 01.12. Aufzeichnung des Rechtsberaters Kaufmann

Kaufmann erörtert die Überlegungen der AHK vom 11. November $1953 \mathrm{zu}$ freien gesamtdeutschen Wahlen und zu den Befugnissen einer gesamtdeutschen Regierung.

$348 \quad$ 01.12. Staatssekretär Hallstein, z.Z. Washington, an Bundeskanzler Adenauer

Hallstein informiert über Gespräche mit dem amerikanischen Außenminister Dulles. Im Mittelpunkt standen die geplante Vier-Mächte-Konferenz und die Ratifizierung des EVG-Vertrags durch die französische Nationalversammlung.

Erläutert wird der Stand der Arbeiten der interministeriellen Arbeitsgruppen, die im August 1953 zur Vorbereitung einer Vier-Mächte-Konferenz gebildet wurden.

350 03.12. Ministerialdirigent Bräutigam an die Botschaft in Athen

Aufgrund von Berichten über Vorstöße der DDR zur Errichtung eines Handelsbüros in Griechenland legt Bräutigam die Haltung der Bundesregierung dar.

Amt

Krekeler gibt Informationen über den Stand der Überlegungen im amerikanischen Außenministerium zu einer Vier-MächteKonferenz und zu einer möglichen Reduzierung der amerikanischen Streitkräfte in Europa.

Amt

Koenning berichtet über ein Gespräch mit dem finnischen AuBenminister Törngren, in dem die finnische Haltung zur Bundesrepublik und zur DDR sowie Wirtschaftsfragen erörtert wurden. diplomatische Vertretung in London

Maltzan informiert über die Übermittlung eines Schreibens des Bundeskanzlers Adenauer an Premierminister Churchill zur geplanten Vier-Mächte-Konferenz. 
Erörtert werden die Vorschläge der AHK vom 11. November 1953 zu gesamtdeutschen Wahlen und zu den Befugnissen einer daraus hervorgehenden gesamtdeutschen Regierung.

Hausenstein berichtet über eine Unterredung mit dem Abteilungsleiter im französischen Außenministerium, de la Tournelle, in der die Frage einer Freilassung der im Oradour-Prozeß verurteilten Deutschen besprochen wurde.

09.12. Gespräch des Staatssekretärs Hallstein mit dem

französischen Stellvertretenden Hohen Kommissar

Bérard

Die Gesprächspartner erörtern einen „accord de principe“ zur Saarfrage.

Hallstein erläutert die Haltung der Bundesregierung zur bevorstehenden Vier-Mächte-Konferenz und den Stand der Saargespräche. Er befaßt sich zudem mit der Ratifizierung des EVGVertrags in Frankreich.

Amt

Krekeler gibt Äußerungen von Mitarbeitern des amerikanischen Außenministeriums zur Saarfrage wieder und bittet um weitere Sprachregelung. Amt

Oellers berichtet über Sondierungen der DDR mit dem Ziel, wirtschaftliche Beziehungen zu Brasilien aufzunehmen.

Erörtert werden Fragen, die zwischen der Bundesrepublik und den Niederlanden ungelöst sind. amerikanischen Außenminister Dulles in Paris

Themen sind die Ratifizierung des EVG-Vertrags in Frankreich und Italien, die Saarfrage, die bevorstehende Vier-Mächte-Konferenz und die Präsenz amerikanischer Truppen in Europa. 

britischen Außenminister Eden in Paris

Adenauer und Eden erörtern die bevorstehende Vier-MächteKonferenz, insbesondere den britisch-französischen Entwurf für eine Sicherheitserklärung, die EVG und die Vorschläge des belgischen Außenministers van Zeeland zur europäischen Sicherheit.

Frank resümiert ein Gespräch mit Bundeskanzler Adenauer über die Frage, mit welchen Mitteln sich die Aussichten auf eine Ratifizierung des EVG-Vertrags in Frankreich fördern ließen.

Der Kabinettsausschuß für die Vier-Mächte-Konferenz befaßt sich mit Möglichkeiten zur Durchführung freier gesamtdeutscher Wahlen.

Salat informiert über eine Ressortbesprechung, die sich mit der Zuständigkeit für die Europäische Organisation für kernphysikalische Forschung und mit der Beschaffung der dafür erforderlichen finanziellen Mittel befaßte.

16.12. Besprechung mit Vertretern der Alliierten Hohen Kommission

Rechtsberater Kaufmann erläutert den Vertretern der AHK die Haltung der Bundesregierung zu den Befugnissen einer gesamtdeutschen Regierung und zur Kontrolle gesamtdeutscher Wahlen. Hallstein

Krekeler äußert sich zu Reaktionen in den USA auf den Besuch des Bundesministers Erhard. Abteilungsleiter Grewe in Paris

Erörtert wird der von Abteilungsleiter Grewe dargelegte Standpunkt der Bundesregierung zur Durchführung gesamtdeutscher Wahlen und zu den Befugnissen einer gesamtdeutschen Regierung.

Etzdorf berichtet von einem Gespräch mit dem Vorsitzenden des Interimsausschusses der EVG-Konferenz, Alphand, über die amerikanische Haltung zur EVG. 

Abteilungsleiter Grewe in Paris

Die Sachverständigen befassen sich mit den Befugnissen einer provisorischen gesamtdeutschen Regierung. Auswärtige Amt

Schmidt-Schlegel berichtet über eine Unterredung mit dem Staatssekretär im bolivianischen Außenministerium, Quiroga Galdo. Im Mittelpunkt standen die Wirtschaftsbeziehungen und eine Aufwertung der diplomatischen Vertretungen zu Botschaften.

In einer Auseinandersetzung mit dem Bundesministerium für wirtschaftliche Zusammenarbeit ersucht Hermes um Unterstützung für seine Verhandlungsführung bei der Konferenz für die Organisation der Europäischen Agrarmärkte. Abteilungsleiter Grewe in Paris

Diskutiert werden die Durchführung freier gesamtdeutscher Wahlen und die Befugnisse einer gesamtdeutschen Regierung.

Kaufmann erörtert die Frage, unter welchen Voraussetzungen eine gesamtdeutsche Regierung mit allen Machtbefugnissen auszustatten sei und zu welchem Zeitpunkt Friedensverhandlungen beginnen sollten.

Salat befaßt sich mit der Frage der Zustimmungsbedürftigkeit von Kulturabkommen mit dritten Staaten durch die Länder.

Kessel stellt Überlegungen zu gesamtdeutschen Wahlen und zur Übernahme der staatlichen Vollmachten durch eine gesamtdeutsche Nationalversammlung und Regierung an.

Hallstein übermittelt eine Sprachregelung zum Vorschlag des belgischen Außenministers van Zeeland für ein europäisches Sicherheitssystem. 
Ophüls nimmt zum Schreiben des Delegationsleiters Hermes über die Verhandlungsführung auf der Konferenz für die Organisation der Europäischen Agrarmärkte Stellung.

Welck äußert sich zur österreichischen Absicht, die Initiative zur Errichtung einer diplomatischen Vertretung der Bundesrepublik in Wien zu ergreifen.

Hallstein bittet darum, östliche Bestrebungen zu beobachten, der DDR zur staatlichen Anerkennung zu verhelfen.

Melchers berichtet über Gespräche mit der jordanischen Regierung anläßlich der Übergabe des Beglaubigungsschreibens in Amman. Im Mittelpunkt standen der Israel-Vertrag, die Wirtschaftsbeziehungen und Sichtvermerksfragen.

Welck befaßt sich mit den von der UdSSR auf der Vier-MächteKonferenz zu erwartenden Vorschlägen und entwirft Stellungnahmen der Bundesregierung dazu.

31.12. Vizekonsul Löwe, Berlin (West), an das Auswärtige Amt

Löwe informiert über Erwartungen der SED im Hinblick auf die Vier-Mächte-Konferenz in Berlin. 


\section{Literaturverzeichnis}

AAPD

AAPD, Hohe Kommissare 1952

ACTES DE L'UNION POSTALE

ADAP, A, III

$\mathrm{ADAP}, \mathrm{B}, \mathrm{II}$

ADAP, D, II

ADENAUER, Briefe 1949-1951

ADENAUER, Briefe 1951-1953

ADENAUER, Briefe 1953-1955

ADENAUER, Erinnerungen 1945-1953

ADENAUER, Erinnerungen 1953-1955
Akten zur Auswärtigen Politik der Bundesrepublik Deutschland, hrsg. im Auftrag des Auswärtigen Amts vom Institut für Zeitgeschichte. Jahresband 1949/50. Jahresband 1951. Jahresband 1952, München 1997, 1999 und 2000.

Akten zur Auswärtigen Politik der Bundesrepublik Deutschland. Band 2: Adenauer und die Hohen Kommissare 1952, hrsg. von Hans-Peter Schwarz in Verbindung mit Reiner Pommerin, München 1990.

Les Actes de l'Union postale universelle révisés à Paris 1947 et annotés par les soins du Bureau international, hrsg. vom Bureau international de l'Union postale universelle, Bern 1949.

Akten zur deutschen auswärtigen Politik 19181945. Serie A (1918-1925). Band III: 1. Januar bis 30. September 1920, Göttingen 1985.

Akten zur deutschen auswärtigen Politik 19181945. Serie B (1925-1933). Band II: Deutschlands Beziehungen zur Sowjet-Union, zu Polen, Danzig und den Baltischen Staaten (Dezember 1925-Dezember 1926), 2 Halbbände, Göttingen 1967.

Akten zur deutschen auswärtigen Politik 19181945. Serie D (1937-1945). Band II: Deutschland und die Tschechoslowakei (Oktober 1937September 1938), Baden-Baden 1950.

Adenauer. Rhöndorfer Ausgabe: Briefe 19491951, hrsg. von Rudolf Morsey und Hans-Peter Schwarz, bearbeitet von Hans Peter Mensing, Berlin 1985.

Adenauer. Rhöndorfer Ausgabe: Briefe 19511953, hrsg. von Rudolf Morsey und Hans-Peter Schwarz, bearbeitet von Hans Peter Mensing, Berlin 1987.

Adenauer. Rhöndorfer Ausgabe: Briefe 19531955, hrsg. von Rudolf Morsey und Hans-Peter Schwarz, bearbeitet von Hans Peter Mensing, Berlin 1995.

Konrad Adenauer, Erinnerungen 1945-1953, Stuttgart 1965.

Konrad Adenauer, Erinnerungen 1953-1955, Stuttgart 1966. 
ADENAUER-HEUSS

AdG

AD-HOC-VERSAMMLUNG

AMERICAN FOREIGN POLICY 1950-1955

AMTSBLATT DER AHK

AMTSBLATT DER MILITÄRREGIERUNG DEUTSCHLAND (Britisches Kontrollgebiet)

\section{AMTSBLATT DES FRANZÖSISCHEN OBERKOMMANDOS}

AMTSBLATT DES SAARLANDES

AUSSENPOLITIK DER DDR I

AUSWÄRTIGE POLITIK

AUSWÄRTIGER AUSSCHUSS

BDFD I
Adenauer. Rhöndorfer Ausgabe: AdenauerHeuss. Unter vier Augen. Gespräche aus den Gründerjahren 1949-1959, hrsg. von Rudolf Morsey und Hans-Peter Schwarz, bearbeitet von Hans Peter Mensing, Berlin 1995.

Keesing's Archiv der Gegenwart, zusammengestellt von Heinrich von Siegler, Wien $1950 \mathrm{ff}$.

Ad-hoc-Versammlung, beauftragt mit der Ausarbeitung eines Vertragsentwurfes für die Gründung einer Europäischen Politischen Gemeinschaft, Sitzungsperiode März 1953. Band 1: Kurzberichte über die Beratungen; Sitzungsprotokolle, Straßburg 1953.

American Foreign Policy 1950-1955. Basic Documents, 2 Bände, Washington 1957.

Amtsblatt der Alliierten Hohen Kommission in Deutschland. Nr. 1-72 [o.O.] 1949-1951.

Amtsblatt der Militärregierung Deutschland. Nr. 1-3: Kontrollgebiet der 21. Armeegruppe; Nr. 4-28: Britisches Kontrollgebiet; Nr. 29-36: Amtsblatt der Kontrollkommission Deutschland (Britische Zone) [1945-1949].

Amtsblatt des französischen Oberkommandos in Deutschland. Nr. 1-305 [1945-1949].

Amtsblatt des Saarlandes, hrsg. vom Informationsamt der Regierung des Saarlandes, Saarbrücken $1947 \mathrm{ff}$.

Dokumente zur Außenpolitik der Regierung der Deutschen Demokratischen Republik. Band I: Von der Gründung der Deutschen Demokratischen Republik am 7. Oktober 1949 bis zur Souveränitätserklärung am 25. März 1954, hrsg. vom Deutschen Institut für Zeitgeschichte, Berlin [Ost] 1954.

Die Auswärtige Politik der Bundesrepublik Deutschland, hrsg. vom Auswärtigen Amt unter Mitwirkung eines wissenschaftlichen Beirats, Köln 1971.

Der Auswärtige Ausschuß des Deutschen Bundestages. Sitzungsprotokolle 1949-1953. 1. Halbband: Oktober 1949 bis Mai 1952; 2. Halbband: Juni 1952 bis August 1953 und Unterausschuß „Besatzungsregime“ 1951/52, bearbeitet von Wolfgang Hölscher, Düsseldorf 1998.

Die Bundesrepublik Deutschland und Frankreich. Dokumente 1949 bis 1963. Band 1: Außenpolitik und Diplomatie, hrsg. von Horst Möller und Klaus Hildebrand, bearbeitet von Ulrich Lappenküper, München 1997. 
BDFD II

BEENDIGUNG DES KRIEGSZUSTANDS

BEMÜHUNGEN I

BERARD, Ambassadeur

BERLIN 1951-1954

BEZIEHUNGEN ZUM HeILIGEN STUHL

BLANKENHORN, Verständnis

BR DRUCKSACHEN

BR SITZUNGSBERICHTE

BT ANLAGEN

BT STENOGRAPHISCHE BERICHTE

BULLETIN

BUNDESANZEIGER
Die Bundesrepublik Deutschland und Frankreich: Dokumente 1949 bis 1963. Band 2: Wirtschaft, hrsg. von Horst Möller und Klaus Hildebrand, bearbeitet von Andreas Wilkens, München 1997.

Die Beendigung des Kriegszustands mit Deutschland nach dem Zweiten Weltkrieg, bearbeitet von Hermann Mosler und Karl Doehring, Köln/Berlin 1963.

Die Bemühungen der Bundesrepublik um Wiederherstellung der Einheit Deutschlands durch gesamtdeutsche Wahlen. Dokumente und Akten. I. Teil: Oktober 1949-Oktober 1953, hrsg. vom Bundesministerium für gesamtdeutsche Fragen, 4. erweiterte Auflage, Bonn 1953.

Armand Bérard, Un Ambassadeur se souvient. Band 2: Washington et Bonn 1945-1955, Paris 1978.

Berlin. Chronik der Jahre 1951-1954, hrsg. im Auftrage des Senats von Berlin, bearbeitet von Hans J. Reichhardt, Joachim Drogmann und Hanns U. Treutler, Berlin 1968.

Die Beziehungen der Bundesrepublik Deutschland zum Heiligen Stuhl 1949-1966. Aus den Vatikanakten des Auswärtigen Amts. Eine Dokumentation, hrsg. von Michael F. Feldkamp, Köln/Weimar/Wien 2000.

Herbert Blankenhorn, Verständnis und Verständigung. Blätter eines politischen Tagebuchs 1949 bis 1979, Frankfurt am Main/Berlin/Wien 1980 .

Deutscher Bundesrat. Drucksachen, Bonn $1949 \mathrm{ff}$.

Deutscher Bundesrat. Sitzungsberichte, Bonn $1949 \mathrm{ff}$.

Verhandlungen des Deutschen Bundestages. Anlagen $\mathrm{zu}$ den Stenographischen Berichten, Bonn 1949 ff.

Verhandlungen des Deutschen Bundestages. Stenographische Berichte, Bonn 1949 ff.

Bulletin des Presse- und Informationsamtes der Bundesregierung, Bonn 1952 ff.

Bundesanzeiger (bis August 1950: Bundesanzeiger und Offentlicher Anzeiger für das Vereinigte Wirtschaftsgebiet. Bekanntmachungsorgan für das französische Besatzungsgebiet. Verkündungsorgan für die Verlautbarungen des Einfuhrausschusses), hrsg. vom Bundesminister der Justiz, Bonn 1949 ff. 


\section{BUNDES-GESETZBLATT DES NORDDEUTSCHEN BUNDES 1867 \\ BUNDESGESETZBLATT}

CDU/CSU-FRAKTION

Charter of THE UNited Nations

ChURCHILL, Second World War

Churchill, Post-War Speeches

CONGRESSIONAL RECORD

CONSTITUTIONS OF NATIONS

COUNCIL OF EUROPE, CONSUlTATIVE ASSEMBLY 1953, DEBATES

Council of Europe, Consultative ASSEMBLY 1952, DOCUMENTS

Council of Europe, Consultative ASSEMBLY 1953, DOCUMENTS

Council of Europe, Consultative ASSEMBLy 1953, TEXTS

Council of Europe, Ministers 1953, DOCUMENTS
Bundes-Gesetzblatt des Norddeutschen Bundes, Berlin 1867.

Bundesgesetzblatt, hrsg. vom Bundesminister der Justiz, Köln 1949 ff.

Die CDU/CSU-Fraktion im Deutschen Bundestag 1949-1966. 1. Halbband: Sitzungsprotokolle 1949-1953, bearbeitet von Helge Heidemeyer, Düsseldorf 1998.

Charter of the United Nations. Commentary and Documents, hrsg. von Leland M. Goodrich, Edvard Hambro und Anne Patricia Simons, 3. überarbeitete Auflage, New York/London 1969.

Winston S. Churchill, The Second World War. Band VI: Triumph and Tragedy, London u.a. 1954.

The Collected Works of Sir Winston Churchill. Centenary Limited Edition. Band XXVIII: PostWar Speeches. Band 1: The Sinews of Peace; Europe Unite, London 1975.

Congressional Record. Proceedings and Debates of the 82nd Congress, Second Session. Band 98, Teil 1 (January 8, 1952 to February 25, 1952); Proceedings and Debates of the 83rd Congress, First Session. Band 99, Teil 2 (February 26, 1953, to April 8, 1953), hrsg. vom United States Government Printing Office, Washington D.C. 1952 und 1953.

Amos J. Peaslee, Constitutions of Nations. Revised Third Edition in Four Volumes. Band II: Asia, Australia and Oceania; Band III: Europe; Band IV: The Americas, Den Haag 1966, 1968 und 1970.

Council of Europe. Consultative Assembly. Fifth Ordinary Session. Official Report of Debates. 5 Bände, Straßburg 1953.

Council of Europe. Consultative Assembly. Fourth Ordinary Session. Documents. Working Papers, 3 Bände, Straßburg 1952.

Council of Europe. Consultative Assembly. Fifth Ordinary Session. Documents. Working Papers, 5 Bände, Straßburg 1953.

Council of Europe. Consultative Assembly. Fifth Ordinary Session. Texts Adopted by the Assembly, Straßburg 1953.

Council of Europe. Documents of the Committee of Ministers 1953-I (January-June, 1953); II (July-December, 1953), Straßburg 1953. 
COUNCIL OF EuRoPe, Ministers, RESOLUTIONS 1949-1951

DBFP 1, XXV

DEPARTMENT OF STATE BULLETIN

DEUTSChe AusLandsschuldEN

DEUTSCHES VERMÖGEN IM AUSLAND

DIPLOMATISCHER KURIER 1953

DOCUMENTS. COMPANION VOLUME 1953

\section{DOCUMENTS ON AMERICAN FOREIGN RELATIONS 1953}

DOCUMENTS ON INTERNATIONAL AFFAIRS 1936

DOCUMENTS ON INTERNATIONAL AFFAIRS 1952

DOCUMENTS ON INTERNATIONAL AFFAIRS 1953
Council of Europe. Resolutions of the Committee of Ministers. First to the Ninth Session 19491951, Straßburg 1952.

Documents on British Foreign Policy 19191939. Serie 1, Band XXV: Russia 1923-1925; Poland an the Baltic States 1924/1925, hrsg. von W.N. Medlicott und Douglas Dakin unter Mitarbeit von Gillian Bennett, London 1984.

The Department of State Bulletin. The Official Weekly Record of United States Foreign Policy, Washington D.C. $1939 \mathrm{ff}$.

Deutsche Auslandsschulden. Dokumente zu den internationalen Verhandlungen, Oktober 1950 bis Juli 1951, hrsg. vom Auswärtigen Amt, dem Bundesministerium für Wirtschaft und dem Bundesministerium für den Marshallplan, Hameln [1951].

Deutsches Vermögen im Ausland. Internationale Vereinbarungen und ausländische Gesetzgebung. Mit Unterstützung des Bundesministeriums der Finanzen, des Bundesministeriums für Wirtschaft, des Bundesministeriums für den Marshallplan und der Bank deutscher Länder hrsg. vom Bundesministerium der Justiz, bearbeitet von Otto Böhmer, Konrad Duden und Hermann Jansen, Köln 1951.

Diplomatischer Kurier in Verbindung mit Diplomatische Korrespondenz, Köln 1953.

Documents on the Foreign Policy of Israel. Band 8: 1953 Companion Volume, hrsg. von Yemima Rosenthal, Jerusalem 1995.

Documents on American Foreign Relations 1953, hrsg. von Peter V. Curl im Auftrag des Council on Foreign Relations, New York 1954.

Documents on International Affairs 1936. Issued under the auspicies of the Royal Institute of International Affairs, hrsg. von Stephen Heald in Verbindung mit John W. WheelerBennett, London 1937.

Documents on International Affairs 1952. Issued under the auspicies of the Royal Institute of International Affairs, ausgewählt und hrsg. von Denise Folliot, London/New York/Toronto 1955.

Documents on International Affairs 1953. Issued under the auspicies of the Royal Institute of International Affairs, ausgewählt und hrsg. von Denise Folliot, London/New York/Toronto 1956. 


\section{DOKUMENT VON GROSSER HISTORISCHER BEDEUTUNG}

DOKUMENTE ZUR DEUTSCHEN VERFASSUNGSGESCHICHTE

DOKUMENTE ZUR DEUTSCHLANDPOLITIK DER SOWJETUNION I

DOKUMENTE DES GETEILTEN DEUTSCHLAND

\section{DOKUMENTY I MATERIAEY}

DOKUMENTY VNEŠNEJ POLITIKI SSSR

DROIT INTERNATIONAL

DYNAMICS OF WORLD POWER

DzD I/3

DZD II/1
Rolf Stöckigt, Ein Dokument von großer historischer Bedeutung vom Mai 1953, in: Beiträge zur Geschichte der Arbeiterbewegung 32 (1990), S. 648-654.

Dokumente zur deutschen Verfassungsgeschichte. Band 1: Deutsche Verfassungsdokumente 1803-1850, hrsg. von Ernst Rudolf Huber, 3. neubearbeitete und vermehrte Auflage, Stuttgart u. a. 1978.

Dokumente zur Deutschlandpolitik der Sowjetunion. Band I: Vom Potsdamer Abkommen vom 2. August 1945 bis zur Erklärung über die Herstellung der Souveränität der Deutschen Demokratischen Republik am 25. März 1954, hrsg. vom Deutschen Institut für Zeitgeschichte, Berlin [Ost] 1957.

Dokumente des geteilten Deutschland. Quellentexte zur Rechtslage des Deutschen Reiches, der Bundesrepublik Deutschland und der Deutschen Demokratischen Republik. Band 1, hrsg. von Ingo von Münch, 2. Auflage, Stuttgart 1976.

Dokumenty i materiały do historii stosunków polsko-radzieckich, hrsg. von der Polska Akademia Nauk, Instytut Krajów Socjalistycznych, und der Akademia Nauk ZSRR, Instytut Słowianoznawstwa i Bałkanystiki. Bd. VIII: styczeń 1944-grudzień 1945, bearbeitet von Euzebiusz Basiński u. a., Warschau 1974.

Dokumenty vnešnej politiki SSSR, hrsg. vom Ministerstvo inostrannych del SSSR. Bd. 1 ( 7 nojabrja 1917 g.-31 dekabrja 1918 g.), Moskau 1957.

Droit International et Histoire Diplomatique. Documents choisis par Claude-Albert Colliard, 2. Auflage, Paris 1950.

The Dynamics of World Power. A Documentary History of United States Foreign Policy 19451973, hrsg. von Arthur M. Schlesinger, Band II: Eastern Europe and the Soviet Union, hrsg. von Walter LaFeber, New York 1973.

Dokumente zur Deutschlandpolitik. I. Reihe: Vom 3. September 1939 bis 8 . Mai 1945. Band 3: 1. Januar bis 31. Dezember 1942. Britische Deutschlandpolitik, 2 Teilbände, hrsg. vom Bundesministerium für innerdeutsche Beziehungen, bearbeitet von Rainer A. Blasius, Frankfurt am Main 1989.

Dokumente zur Deutschlandpolitik. II. Reihe: Vom 9. Mai 1945 bis 4. Mai 1955. Band 1: Die Konferenz von Potsdam, 3 Teilbände, hrsg. vom 
DzD II/2

DzD II/3

EUROPA-ARCHIV

Fall BeriJa

FRUS 1948

FRUS 1949

FRUS 1951

FRUS 1952-1954

DE GaUlle, Discours et Messages

GERMANY 1947-1949
Bundesminister des Innern, bearbeitet von $\mathrm{Gi}$ sela Biewer, Neuwied/Frankfurt am Main 1992.

Dokumente zur Deutschlandpolitik. II. Reihe: Vom 9. Mai 1945 bis 4. Mai 1955. Band 2: Die Konstituierung der Bundesrepublik Deutschland und der Deutschen Demokratischen Republik 7. September bis 31. Dezember 1949, hrsg. vom Bundesministerium des Innern unter Mitwirkung des Bundesarchivs, bearbeitet von Hanns Jürgen Küsters unter Mitarbeit von Daniel Hoffmann, München 1996.

Dokumente zur Deutschlandpolitik. II. Reihe: Vom 9. Mai 1945 bis 4. Mai 1955. Band 3: 1. Januar bis 31. Dezember 1950, hrsg. vom Bundesministerium des Innern unter Mitwirkung des Bundesarchivs, bearbeitet von Hanns Jürgen Küsters, Daniel Hoffmann und Carsten Tessmer, München 1997.

Europa-Archiv. Zeitschrift für Internationale Politik, Frankfurt am Main 1946 ff.

Der Fall Berija. Protokoll einer Abrechnung. Das Plenum des ZK der KPdSU, Juli 1953. Stenographischer Bericht, hrsg. und aus dem Russischen übersetzt von Viktor Knoll und Lothar Köhn, Berlin 1993.

Foreign Relations of the United States, 1948. Band II: Germany and Austria, Washington D.C. 1973.

Foreign Relations of the United States, 1949. Band III: Council of Foreign Ministers; Germany and Austria, Washington D.C. 1974.

Foreign Relations of the United States, 1951. Band I: National Security Affairs; Foreign Economic Policy; Band III: European Security and the German Question, 2 Teilbände, Washington D.C. 1979 und 1981.

Foreign Relations of the United States, 19521954. Band V: Western European Security, 2 Teilbände; Band VI: Western Europe and Canada, 2 Teilbände; Band VII: Germany and Austria, 2 Teilbände, Washington D.C. 1983 und 1986

Charles de Gaulle, Discours et Messages. Band 2: Dans l'attente (Février 1946-avril 1958), [Paris] 1970.

Germany 1947-1949. The Story in Documents, hrsg. vom Department of State, Washington D.C. 1950 . 
GeSamtdeutscher Ausschuss

\section{GESETZBLATT DER DDR \\ GESETZBLATT DER VERWALTUNG DES VEREINIGTEN WIRTSCHAFTS- GEBIETES 1947-1949}

GESETZ- UND VERORDNUNGSBLATT FÜR SCHLESWIG-HOLSTEIN 1949

GROSSE POLITIK

GUGGENHEIM, Lehrbuch des Völkerrechts

HANSARD, COMMONS

HANSARD, LORDS

HERRNSTADT-DOKUMENT

JAKOB KAISER

JOURNAL OfFiciel, ASSEMblée NATIONALE 1953 JOURNAL OFFICIEL, CONSEIL DE LA
RÉPUBLIQUE 1953

JOURNAL OFFICIEL, LOIS ET DÉCRETS
Der Gesamtdeutsche Ausschuß. Sitzungsprotokolle des Ausschusses für gesamtdeutsche Fragen des Deutschen Bundestages 1949-1953, bearbeitet von Andreas Biefang, Düsseldorf 1998.

Gesetzblatt der Deutschen Demokratischen Republik, Berlin [Ost] $1949 \mathrm{ff}$.

Gesetzblatt der Verwaltung des Vereinigten Wirtschaftsgebietes 1947-1949, hrsg. vom Büro des Wirtschaftsrates, Frankfurt am Main [o.J.].

Gesetz- und Verordnungsblatt für SchleswigHolstein. Jahrgang 1949, Kiel 1949.

Die Große Politik der europäischen Kabinette 1871-1914. 5. Band: Neue Verwickelungen im Osten 1885-1887, im Auftrage des Auswärtigen Amtes hrsg. von Johannes Lepsius, Albrecht Mendelssohn Bartholdy und Friedrich Thimme, Berlin 1926.

Paul Guggenheim, Lehrbuch des Völkerrechts. Unter Berücksichtigung der internationalen und schweizerischen Praxis, 2 Bände, Basel 1948 und 1951.

Parliamentary Debates (Hansard). House of Commons, Official Report. Fifth Series. Band 507-518 (Session 1952/1953), London 1953.

The Parliamentary Debates (Hansard). House of Lords, Official Report. Fifth Series. Band 179-183 (Session 1952/1953), London 1953.

Rudolf Herrnstadt, Das Herrnstadt-Dokument. Das Politbüro der SED und die Geschichte des 17. Juni 1953, hrsg., eingeleitet und bearbeitet von Nadja Stulz-Herrnstadt, Hamburg 1990.

Jakob Kaiser: Gewerkschafter und Patriot. Eine Werkauswahl, hrsg. und eingeleitet von Tilman Mayer, Köln 1988.

Journal Officiel de la République Française. Débats Parlementaires. Assemblée Nationale. Compte rendue in extenso des séances. Questions écrites et réponses des Ministres à ces questions, Paris 1953.

Journal Officiel de la République Française. Débats Parlementaires. Conseil de la République. Compte rendue in extenso des séances. Questions écrites et réponses des Ministres à ces questions, Paris 1953.

Journal Officiel de la République Française. Lois et décrets, arrêtés, circulaires, avis, communications, informations et annonces. Jahr- 
17. JUNI 1953

KABINETTSAUSSCHUSS FÜR WIRTSCHAFT

KABINETTSPROTOKOLLE

KAPS, Presse

KONKORDATE

LAWS OF THE STATE OF ISRAEL

LENZ, Zentrum

MACHTÜBERNAHME

MONITEUR OFFICIEL

NATO FINAL COMMUNIQUES gang 1948, 1952 und 1953, Paris 1948, 1952 und 1953 .

17. Juni 1953. Arbeiteraufstand in der DDR, hrsg. von Ilse Spittmann und Karl Wilhelm Fricke, Köln 1982.

Die Kabinettsprotokolle der Bundesregierung, hrsg. für das Bundesarchiv von Friedrich $\mathbf{P}$. Kahlenberg. Kabinettsausschuß für Wirtschaft. Band 1: 1951-1953, bearbeitet von Ulrich Enders, München 1999.

Die Kabinettsprotokolle der Bundesregierung, hrsg. für das Bundesarchiv von Hans Booms. Band 3: 1950. Wortprotokolle, bearbeitet von Ulrich Enders und Konrad Reiser; Band 5: 1952 , bearbeitet von Kai von Jena; Band 6 : 1953, bearbeitet von Ulrich Enders und Konrad Reiser, Boppard am Rhein 1986 und 1989.

Paul Kaps, Die Presse ist an allem schuld. Begegnungen eines pfälzischen Zeitungsmannes, Neustadt an der Weinstraße 1974.

Konkordate seit 1800 . Originaltext und deutsche Übersetzung der geltenden Konkordate, zusammengestellt und bearbeitet von Dr. jur. Lothar Schöppe, Frankfurt am Main/Berlin 1964.

Laws of the State of Israel. Band 4: 5710 1949/50. From 22nd Cheshvan, 5710-14.11.49 to 27th AV, 5710-10.8.50. Authorised Translation from the Hebrew, Prepared at the Ministry of Justice, o. O., o.J.

Im Zentrum der Macht. Das Tagebuch von Staatssekretär Lenz 1951-1953, bearbeitet von Klaus Gotto, Hans-Otto Kleinmann und Reinhard Schreiner, Düsseldorf 1989.

Boris Meissner, Die kommunistische Machtübernahme in den baltischen Staaten. Dokumentation, in: Vierteljahrshefte für Zeitgeschichte 2 (1954), S. 95-114.

République Française. Secrétariat d'Etat aux Affaires Economiques, Moniteur Officiel du Commerce et de l'Industrie. Organe Officiel du Centre National du Commerce Exterieur, Paris 1952.

Texts of Final Communiques 1949-1974. Issued by Ministerial Sessions of the North Atlantic Council, the Defence Planning Committee, and the Nuclear Planning Group, Brüssel o. J. 
NATO-HANDBUCH

NOUVEAU RECUEIL DE TRAITÉS

ÖSTERREICHISCHES RECHT

OST-PROBLEME

II. PARTEIKONFERENZ

PFLEIDERER, Politik

POLITISCHE VERTRÄGE

PREUSSisChe GeSETZSAMmLUNG 1869

Public PAPERS, EISENhower 1953

Public PaPers, RoOSEVElT 19281932
NATO-Handbuch, hrsg. vom InformationsDienst der Nordatlantikpakt-Organisation, München [1952].

Nouveau Recueil de Traités d'Alliance, de Paix, de Trève, de Neutralité, de commerce, de limites, d'échange etc. et de plusieurs autres actes servant à la connaissance des relations étrangères des Puissances et états de l'Europe tant dans leur rapport mutuel que dans celui envers les puissances et états dans d'autres parties du globe. Depuis 1808 jusqu'à présent. Tiré des copies publiées par autorité, des meilleures collections particulières de traités et des auteurs les plus estimés par George Frédéric de Martens. Band III: 1808-1818 inclusiv; Band VII: 1820-1827 inclusiv, Göttingen 1877 und 1882 (Reprint).

Österreichisches Recht. Textausgabe österreichischer Gesetze, Verordnungen und Erlässe in einem Band, hrsg. von Dr. Gottfried Andreas und Dr. Erwin Guttenfeld, 5. erweiterte Auflage nach dem Stande vom 1. Juli 1952, Wien 1953.

Ost-Probleme, hrsg. von der Information Division, HICOG, Jahrgang 1952. Jahrgang 1953, Bad Nauheim 1952 und 1953.

Protokoll der Verhandlungen der II. Parteikonferenz der Sozialistischen Einheitspartei Deutschlands, 9. Bis 12. Juli 1952 in der Werner-Seelenbinder-Halle zu Berlin, Berlin [Ost] 1952.

Karl Georg Pfleiderer, Politik für Deutschland. Reden und Aufsätze 1948-1956, Stuttgart 1961.

Politische Verträge. Eine Sammlung von Urkunden. Band 1: Garantiepakte, Bündnisse, Abkommen über politische Zusammenarbeit, Nichtangriffs- und Neutralitätsverträge der Nachkriegszeit, hrsg. von Viktor Bruns, bearbeitet von Georg von Gretschaninow, Berlin 1936.

Gesetz-Sammlung für die Königlichen Preußischen Staaten, Berlin 1869.

Public Papers of the Presidents of the United States. Dwight D. Eisenhower. Containing the Public Messages, Speeches and Statements of the President, January 1 to December 31, 1953, Washington D. C. 1960.

The Public Papers and Addresses of Franklin D. Roosevelt with a Special Introduction and Explanatory Notes by President Roosevelt. 
Public PAPERS, TRUMAN 1949

LES RAPPORTS MENSUELS

REICHS-GESETZBLATT 1871

REICHSGESETZBLATT

REUTER, Schriften-Reden

SCHAUPLATZ BALTIKUM

SCHUTZ, Deutschland

STALIN, Werke

SURVEY OF INTERNATIONAL AFFAIRS 1924

TRIAL OF THE MAJOR WAR CRIMINALS UN GENERAL ASSEMBLy, EIGHTH
SESSION, ANNEXES

UN GENERAL ASSEMBLY, EIGHTH SESSION, FIRST COMMITTEE

UN GENERAL ASSEMBLY, SIXTH SESSION, ANNEXES
Band 1: The Genesis of the New Deal 19281932, New York 1938.

Public Papers of the Presidents of the United States. Harry S. Truman. Containing the Public Messages, Speeches, and Statements of the President, January 1, 1949 to December 31, 1949, Washington D. C. 1964.

Les Rapports Mensuels d'André FrançoisPoncet, Haut-Commissaire français en Allemagne 1949-1955, bearbeitet von Hans Manfred Bock, 2 Bände, Paris 1996.

Reichs-Gesetzblatt, Berlin 1871.

Reichsgesetzblatt, hrsg. vom Reichsministerium des Innern, Berlin 1919-1945.

Ernst Reuter, Schriften-Reden. Band 4: RedenArtikel-Briefe 1949 bis 1953 , bearbeitet von Hans J. Reichhardt, Berlin 1975.

Schauplatz Baltikum. Szenarium einer Okkupation und Angliederung. Dokumente 1939/1940, hrsg., eingeleitet und übersetzt von Michael Rosenbusch, Horst Schützler und Sonja Striegnitz, Berlin 1991.

Wilhelm Wolfgang Schütz, Deutschland am Rande zweier Welten. Voraussetzungen und Aufgaben unserer Außenpolitik, Stuttgart 1952.

J.W. Stalin, Werke. Band 7: 1925; Band 10: 1927. August-Dezember, Berlin [Ost] 1952 und 1953.

Arnold J. Toynbee, Survey of International Affairs 1924, London 1928.

Trial of the Major War Criminals before the International Military Tribunal (Nuremberg 14 November 1945-1 October 1946). Band XXII: Proceedings 27 August 1946-1 October 1946, Nürnberg 1948.

United Nations. Official Records of the General Assembly. Eighth Session. Annexes, 15 September-9 December 1953, New York.

United Nations. Official Records of the General Assembly, Eighth Session. First Committee, Political and Security Questions (Including Regulation of Armaments), Summary Records of Meetings 16 September to 7 December 1953, New York 1953.

United Nations. Official Records of the General Assembly. Sixth Session. Annexes, Paris, 1951-52. 
UN GENERAL ASSEMBLY, SIXTH SESSION, PleNARY MEETINGS

UN SECURITY COUNCIL, SUPPLEMENTS FOR OCTOBER, NOVEMBER AND DECEMBER 1953

UNITED NATIONS RESOLUTIONS I

UNITED NATIONS RESOLUTIONS II

UNITED STATES. STATUTES AT LARGE 1917-1919

UNITED STATES. STATUTES AT LARGE
1946

UNITED STATES. STATUTES AT LARGE 1948

UNITED STATES. STATUTES AT LARGE 1951

UNited STATES. STATUTES AT LARGE 1953

\section{UNTS}

United Nations. Official Records of the General Assembly, Sixth Session. Plenary Meetings, Verbatim Records of Meetings 6 November 1951 to 5 February 1952, Paris 1952.

United Nations. Security Council. Eighth Year. Supplements for October, November and December 1953, New York 1953.

United Nations Resolutions. Serie I: Resolutions Adopted by the General Assembly, hrsg. von Dusan J. Djonovich, New York 1972 ff.

United Nations Resolutions. Serie II: Resolutions and Decisions Adopted by the Security Council, hrsg. von Dusan J. Djonovich, New York $1988 \mathrm{ff}$.

The Statutes at Large of the United States of America from April, 1917, to March, 1919. Concurrent Resolutions of the Two Houses of Congress. Band 40, Washington 1919.

United States. Statutes at Large Containing the Laws and Concurrent Resolutions Enacted during the Second Session of the Seventy-Ninth Congress of the United States of America 1946. Band 60 in 2 Teilbänden, Washington D.C. 1947.

United States. Statutes at Large Containing the Laws and Concurrent Resolutions Enacted during the Second Session of the Eightieth Congress of the United States of America 1948 and Proclamations, Treaties, and International Agreements other than Treaties. Band 62 in 3 Teilbänden, Washington D. C. 1949.

United States. Statutes at Large Containing the Laws and Concurrent Resolutions Enacted during the First Session of the Eighty-Second Congress of the United States of America 1951 and Reorganization Plan, Amendment to the Constitution, and Proclamations. Band 65, Washington D. C. 1952.

United States. Statutes at Large Containing the Laws and Concurrent Resolutions Enacted during the First Session of the Eighty-Third Congress of the United States of America 1953 and Reorganization Plan, Amendment to the Constitution, and Proclamations. Band 67, Washington D.C. 1953.

United Nations Treaty Series. Treaties and International Agreements Registered or Filed and Recorded with the Secretariat of the United Nations, [New York] $1946 \mathrm{ff}$. 
VERFASSUNGSAUSSCHUSS

VNEŚnJAJA POLITIKa SOVETSKOGO SOJUZA

WEHRBEITRAG I

WEHRBEITRAG II

WEHRBEITRAG III

YEARBOOK OF THE UNITED NATIONS
Leitfaden und amtliche Dokumente des Verfassungsausschusses, hrsg. vom Sekretariat des Verfassungsausschusses, Paris 1953.

Vnešnjaja politika Sovetskogo sojuza. 1946 god, [Moskau] 1952.

Der Kampf um den Wehrbeitrag. 1. Halbband: Die Feststellungsklage, hrsg. vom Institut für Staatslehre und Politik e.V., München 1952.

Der Kampf um den Wehrbeitrag. 2. Halbband: Das Gutachtenverfahren (30.7.-15.12.1952), hrsg. vom Institut für Staatslehre und Politik e. V., München 1953.

Der Kampf um den Wehrbeitrag. Ergänzungsband, hrsg. vom Institut für Staatslehre und Politik e.V., München 1958.

Yearbook of the United Nations 1951, 1952 und 1953 hrsg. vom Department of Public Information United Nations, New York 1952, 1953 und 1954. 


\section{Abkürzungsverzeichnis}

AA

Abt.

a. D.

AFP

AG

AGSEC

AHK

AR

A. R.S.

$\mathrm{AZ}$

BBC

Ber. Nr.

BHE

BK

BMF

BMG

BMI

BMJ

BRD

BRM

CDU

CEA

C.E.D.
Auswärtiges Amt

Abteilung

außer Dienst

Agence France Presse

Aktiengesellschaft

Allied General Secretariat

Alliierte Hohe Kommission

Amtsrat

Action Républicaine et

Sociale

Aktenzeichen

British Broadcasting

Corporation

Bericht-Nummer

Bund der

Heimatvertriebenen und

Entrechteten

Bundeskanzler

Bundesminister/ium der Finanzen

Bundesminister/ium für gesamtdeutsche Fragen

Bundesminister/ium des Innern

Bundesminister/ium der Justiz

Bundesrepublik

Deutschland

Bundesminister/ium für

Angelegenheiten des

Bundesrates

Christlich-Demokratische Union Deutschlands

Conféderation Européenne de l'Agriculture

Communauté Européenne de la Défense
CERN

CIA

COCOM

ČSR

$\mathrm{CSU}$

CVP

D

DBD

DDR

Del.

Dg

DINIE

DP

dpa

DPB

DPS

DRK

DSP

ECA

ECE

EDC

EGKS

EPG

ERP
Centre Européen pour la Recherche Nucléaire

Central Intelligence Agency

Coordinating Committee

Československá Republika

Christlich-Soziale Union

Christliche Volkspartei

(Ministerial-)Direktor

Demokratische

Bauernpartei

Deutschlands

Deutsche Demokratische Republik

Delegation

(Ministerial-)Dirigent

Dirección Nacionale Industria del Estado

Deutsche Partei

Deutsche Presseagentur

Deutsche Partei Bayern

Demokratische Partei Saar

Deutsches Rotes Kreuz

Deutsche

Sozialdemokratische Partei

Economic Cooperation

Administration

Economic Commission for

Europe

European Defense

Community

Europäische Gemeinschaft für Kohle und Stahl

Europäische Politische Gemeinschaft

European Recovery Program 


\begin{tabular}{|c|c|c|c|}
\hline EStA & Erster Staatsanwalt & KPdSU & $\begin{array}{l}\text { Kommunistische Partei der } \\
\text { Sowjetunion }\end{array}$ \\
\hline EVG & $\begin{array}{l}\text { Europäische Verteidigungs- } \\
\text { gemeinschaft }\end{array}$ & LDP & Liberal-Demokratische \\
\hline EZU & $\begin{array}{l}\text { Europäische } \\
\text { Zahlungsunion }\end{array}$ & LR & Legationsrat \\
\hline FAO & $\begin{array}{l}\text { Food and Agriculture } \\
\text { Organization }\end{array}$ & $\begin{array}{l}\text { LR I } \\
\text { MB }\end{array}$ & $\begin{array}{l}\text { Legationsrat I. Klasse } \\
\text { Ministerbüro }\end{array}$ \\
\hline FDP & $\begin{array}{l}\text { Freie Demokratische } \\
\text { Partei }\end{array}$ & MD/Min. Dir. & Ministerialdirektor \\
\hline FIFA & $\begin{array}{l}\text { Fédération Internationale } \\
\text { des Football Associations }\end{array}$ & $\begin{array}{l}\text { Mdg/ } \\
\text { Min.Dirig. }\end{array}$ & Ministerialdirigent \\
\hline FU & Föderalistische Union & MG & Maschinengewehr \\
\hline GATT & $\begin{array}{l}\text { General Agreement on } \\
\text { Tariffs and Trade }\end{array}$ & $\begin{array}{l}\text { Mio. } \\
\text { MR/Min. Rat }\end{array}$ & $\begin{array}{l}\text { Million/en } \\
\text { Ministerialrat }\end{array}$ \\
\hline g/Geh./geh. & Geheim/geheim & Mrd. & Milliarde/n \\
\hline GB & Gesamtdeutscher Block & MRP & $\begin{array}{l}\text { Mouvement Républicain } \\
\text { Populaire }\end{array}$ \\
\hline Ges. & Gesandter & MSA & Mutual Security Agency \\
\hline $\begin{array}{l}\text { GG } \\
\text { GK }\end{array}$ & $\begin{array}{l}\text { Grundgesetz } \\
\text { Generalkonsul }\end{array}$ & NATO & $\begin{array}{l}\text { North Atlantic Treaty } \\
\text { Organization }\end{array}$ \\
\hline GR & Gesandtschaftsrat & NDPD & $\begin{array}{l}\text { Nationaldemokratische } \\
\text { Partei Deutschlands }\end{array}$ \\
\hline $\mathrm{HaPol}$ & Klasse & NSDAP & $\begin{array}{l}\text { Nationalsozialistische } \\
\text { Deutsche Arbeiterpartei }\end{array}$ \\
\hline Harol & $\begin{array}{l}\text { Handelspolitische } \\
\text { Abteilung }\end{array}$ & NWDR & $\begin{array}{l}\text { Nordwestdeutscher } \\
\text { Rundfunk }\end{array}$ \\
\hline HICOG & $\begin{array}{l}\text { United States High } \\
\text { Commissioner for Germany }\end{array}$ & Obs. & Observer \\
\hline HICOM & High Commission & OEEC & $\begin{array}{l}\text { Organization for European } \\
\text { Economic Cooperation }\end{array}$ \\
\hline HJ & Hitler-Jugend & ORR & Oberregierungsrat \\
\hline i. A. & im Auftrag & O.T.A.N. & $\begin{array}{l}\text { Organisation du Traité de } \\
\text { l'Atlantique Nord }\end{array}$ \\
\hline IFAP & $\begin{array}{l}\text { International Federation of } \\
\text { Agricultural Producers }\end{array}$ & Reg. Dir. & Regierungsdirektor \\
\hline IMF & $\begin{array}{l}\text { International Monetary } \\
\text { Fund }\end{array}$ & RIAS & $\begin{array}{l}\text { Rundfunk im } \\
\text { amerikanischen Sektor }\end{array}$ \\
\hline i.V. & in Vertretung & $\mathrm{RM}$ & Reichsmark \\
\hline IWF & $\begin{array}{l}\text { Internationaler Währungs- } \\
\text { fonds }\end{array}$ & $\mathrm{RR}$ & Regierungsrat \\
\hline J.-Nr. & Journal-Nummer & R.S & $\begin{array}{l}\text { République Socialiste } \\
\text { Soviétique }\end{array}$ \\
\hline Kab. & Kabinett & SA & Schutzabteilung \\
\hline KPD & $\begin{array}{l}\text { Kommunistische Partei } \\
\text { Deutschlands }\end{array}$ & SACEUR & $\begin{array}{l}\text { Supreme Allied } \\
\text { Commander Europe }\end{array}$ \\
\hline
\end{tabular}




\begin{tabular}{|c|c|c|c|}
\hline SBZ/SBZD & $\begin{array}{l}\text { sowjetische } \\
\text { Besatzungszone } \\
\text { Deutschlands }\end{array}$ & $\begin{array}{l}\text { UK } \\
\text { UN }\end{array}$ & $\begin{array}{l}\text { United Kingdom } \\
\text { United Nations }\end{array}$ \\
\hline SED & $\begin{array}{l}\text { Sozialistische Einheits- } \\
\text { partei Deutschlands }\end{array}$ & UNESCO & $\begin{array}{l}\text { United Nations Educa- } \\
\text { tional, Scientific and } \\
\text { Cultural Organization }\end{array}$ \\
\hline SFIO & $\begin{array}{l}\text { Section française de } \\
\text { l'Internationale ouvrière }\end{array}$ & UNICEF & $\begin{array}{l}\text { United Nations Inter } \\
\text { national Children's }\end{array}$ \\
\hline SHAPE & Supreme Headquarters & & Emergency Fund \\
\hline S.J. & Allied Powers Europe & UNO & $\begin{array}{l}\text { United Nations } \\
\text { Organization }\end{array}$ \\
\hline SKK & $\begin{array}{l}\text { Sowjetische } \\
\text { Kontrollkommission }\end{array}$ & UNRWA & $\begin{array}{l}\text { United Nations Relief and } \\
\text { Works Agency for Palestine } \\
\text { Refugees in the Near East }\end{array}$ \\
\hline SMAD & $\begin{array}{l}\text { Sowjetische } \\
\text { Militäradministration in } \\
\text { Deutschland }\end{array}$ & $\begin{array}{l}\text { UP } \\
\text { U.R.A.S. }\end{array}$ & $\begin{array}{l}\text { United Press } \\
\text { Union des Républicains de }\end{array}$ \\
\hline SPD & $\begin{array}{l}\text { Sozialdemokratische Partei } \\
\text { Deutschlands }\end{array}$ & U.R.S.S. & Union des Républiques \\
\hline SPÖ & $\begin{array}{l}\text { Sozialdemokratische Partei } \\
\text { Österreichs }\end{array}$ & US & Socialistes Soviétiques \\
\hline SS & Schutzstaffel & USA & United States of America \\
\hline SSR & $\begin{array}{l}\text { sozialistische Sowjet- } \\
\text { Republik }\end{array}$ & $\begin{array}{l}\text { VLR } \\
\text { VN }\end{array}$ & $\begin{array}{l}\text { Vortragender Legationsrat } \\
\text { Vereinte Nationen }\end{array}$ \\
\hline StS & Staatssekretär & Vopo & Volkspolizei \\
\hline $\mathrm{SU}$ & Sowjetunion & VS & Verschlußsache \\
\hline TASS & $\begin{array}{l}\text { Telegrafnoe Agentstvo } \\
\text { Sovetskogo Sojuza }\end{array}$ & WPV & Weltpostverein \\
\hline Tgb. Nr. & Tagebuch-Nummer & $\mathrm{Z}$ & Zentrum \\
\hline UdSSR & $\begin{array}{l}\text { Union der Sozialistischen } \\
\text { Sowjetrepubliken }\end{array}$ & $\begin{array}{l}\text { ZK } \\
\text { z. Wv. }\end{array}$ & $\begin{array}{l}\text { Zentralkomitee } \\
\text { zur Wiederverwendung }\end{array}$ \\
\hline
\end{tabular}


